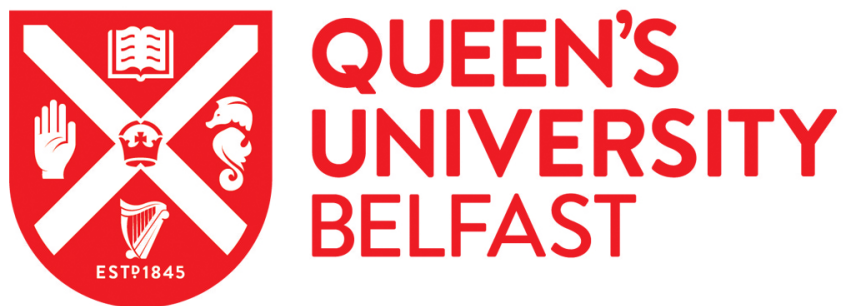

\section{Numerical and experimental validation of an explicit meshfree method: with applications to material forming}

Smith, S. P., Menary, G. H., Nixon, J., \& Falzon, B. G. (2020). Numerical and experimental validation of an explicit meshfree method: with applications to material forming. Engineering Analysis with Boundary Elements, 122, 43-61. https://doi.org/10.1016/j.enganabound.2020.10.013

\section{Published in:}

Engineering Analysis with Boundary Elements

\section{Document Version:}

Peer reviewed version

Queen's University Belfast - Research Portal:

Link to publication record in Queen's University Belfast Research Portal

\section{Publisher rights}

Copyright 2020 Elsevier.

This manuscript is distributed under a Creative Commons Attribution-NonCommercial-NoDerivs License

(https://creativecommons.org/licenses/by-nc-nd/4.0/), which permits distribution and reproduction for non-commercial purposes, provided the author and source are cited.

\section{General rights}

Copyright for the publications made accessible via the Queen's University Belfast Research Portal is retained by the author(s) and / or other copyright owners and it is a condition of accessing these publications that users recognise and abide by the legal requirements associated with these rights.

Take down policy

The Research Portal is Queen's institutional repository that provides access to Queen's research output. Every effort has been made to ensure that content in the Research Portal does not infringe any person's rights, or applicable UK laws. If you discover content in the Research Portal that you believe breaches copyright or violates any law, please contact openaccess@qub.ac.uk. 


\title{
Numerical and experimental validation of an explicit meshfree method: with applications to material forming
}

\author{
Stephen P. Smith, Gary H. Menary, James Nixon, Brian G. Falzon ${ }^{1, *}$ \\ School of Mechanical and Aerospace Engineering, Queen's University Belfast, Belfast BT9 5AH, UK
}

\begin{abstract}
Meshfree methods were introduced twenty-five years ago to overcome a range of issues faced by meshbased methods, which predominately relate to the issues of mesh-entanglement, which can result in poor accuracy. Although meshfree methods have progressed significantly over these years, the application of these techniques to real-world material forming problems is limited, despite the potential benefits they can provide over traditional mesh-based methods. This paper is concerned with the meshfree simulation of the stretch blow moulding process, which is the primary manufacturing process used to produce polymer bottles. Traditionally, the finite element method has been used to simulate this process, however, difficulties are often encountered during these simulations, which can cause reduced accuracy. In this paper, a nodally integration explicit element-free Galerkin method is formulated. Firstly, the formulation is validated through several numerical problems, which display the accuracy, and computational efficiency of the proposed method. Following this, the experimental characterisation of the stretch blow moulding process is discussed. Finally, the developed formulation is utilised to construct a validated simulation of the stretch blow moulding process. The accuracy obtained in the stretch blow moulding simulations highlights the capability of the explicit meshfree formulation, which has the potential to be applied to a wide range of large deformation phenomena.
\end{abstract}

Keywords: Meshfree methods, material forming, explicit dynamics, nodal integration

\section{Introduction}

In recent years the application of numerical methods to forming simulations has grown significantly.

\footnotetext{
*Corresponding author

Email address: b.falzon@qub.ac.uk (Brian G. Falzon) $U R L$ :

https://www.qub.ac.uk/sites/acrg/members/BrianGFalzon/

(Brian G. Falzon)

${ }^{1}$ Advanced Composite Research Group
}

This growth has allowed for the adoption of a scientific approach to process optimisation, rather than the traditional trial and error approach. Of these numerical techniques, the finite element method has emerged as the dominant simulation tool. Although the finite element has proved successful in modelling a range of problems, large deformation can lead to element-distortion. This can cause reduced poly- 
nomial completeness of the elements displacement field, resulting in poor convergence [1]. While techniques such as the smoothed finite element method [2] and suitable remeshing schemes [3, can reduce the sensitivity to mesh distortion, they still encounter the primary issues of requiring the construction of a quality mesh. Creation and refinement of this mesh can be a computationally cumbersome procedure, especially for three-dimensional geometries.

In response to the issues faced by the finite element method in large deformation, a new class of techniques, known as meshfree methods, have been proposed. Numerous techniques have been developed over the years including smoothed particle hydrodynamics, introduced independently by Lucy 4] and Gingold and Monaghan [5], the element-free Galerkin (EFG) method developed by Belytschko et al. [6], and the reproducing kernel particle method (RKPM) Liu et al. [7]. Unlike their mesh-based counterparts, meshfree methods enable the field variable to be approximated without the need for a fixed computational mesh, avoiding mesh related issues such as mesh-distortion and the reliance on a high-quality mesh. These developments have collectively provided an alternative to the finite element method, especially in situations where mesh-based methods can encounter difficulties, such as those involving large deformation.

Typically, meshfree methods are formulated using either the collocation method or a Galerkin method. The former is based on the strong-form of the differential equation, which leads to efficient formulations due to not having to perform numerical integration. However, collocation methods en- counter difficulties in applying natural boundary conditions and require the evaluation of higherorder shape function derivatives, which can be costly to construct [8]. In this work, a Galerkin method is chosen, where a weaker form with regards to solution continuity is solved. This requires the selection of a suitable numerical integration scheme and a technique to implement essential boundary conditions.

In this work, we utilise the element-free Galerkin method, which has primarily been applied in situations where the meshfree characteristic of the method can be exploited. Applications of the EFG method include: fracture and crack growth [9, 10], metal forming simulations [11, 12] and large deformation biomechanics problems [13. In the work of Li and Belytschko [1] several large deformation metal forming problems were simulated. The authors observed that the Lagrangian element-free Galerkin method could deal with larger deformation than the equivalent finite element formulation. The authors attributed this to the smoothness provided by the EFG approximation. A similar finding was observed with the RKPM by Li and Liu [14], where the ability of meshfree methods to simulate large deformation was attributed to the higher continuity of the resulting approximation. Horton et al. 13] formulated an explicit element-free Galerkin formulation for use in surgical simulations, where the reduced dependency on a computational mesh has the potential to aide in automatic model generation. The authors showed though several hyperelastic examples that sufficient levels of accuracy could be achieved. However, the authors also highlighted that the use of Gauss integration leads to 
high computational cost in the resulting formulation, which potentially would require a mixture of EFG and finite methods to be applied.

Given the reported benefits of the EFG method in Lagrangian large deformation simulations, the applicability of this technique to material forming simulations is clear. However, despite these benefits, the use of an EFG method to simulate forming simulations is limited, with [11, 12] showing the capability of the method in high distortion loading, but modest deformations. Furthermore, within this research, rectangular geometries have allowed for simulations with a regular distribution of nodes, which is a condition that cannot always be replicated in an arbitrary forming simulation. This will likely affect the accuracy of the formulation and therefore warrants investigation. Also, the accuracy and computational cost of the explicit EFG formulation has been shown by Horton et al. [13] to be sensitive on the order of integration used, which has the potential to reduce the effectiveness and applicability of the EFG method. Based on these observations, in this work, we aim to fully assess the capability of an explicit EFG method in the presence of both large deformation and large distortion by simulating the polymer forming process stretch blow moulding. Furthermore, in this investigation, we also address the outstanding issues of numerical integration for disordered nodal distributions and its effect on the accuracy and efficiency of the explicit EFG formulation.

Stretch blow moulding (SBM) is the manufacturing process used to produce polymer bottles for the soft drinks industry, with a market estimated to grow to $\$ 46$ billion by 2021 [15]. The manufacturing

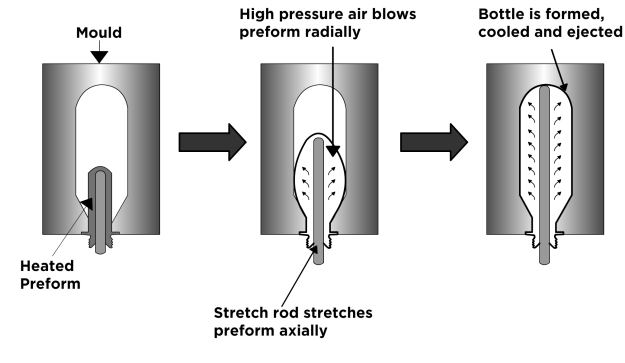

Figure 1: Stretch blow moulding process

process features two primary stages: (1) heating of the preform, constructed from polyethylene terephthalate (PET), above its glass transition temperature $T_{g}$, and (2) axial stretching due to a stretch rod, and radial expansion due to internal pressure within the preform cavity, Figure 1. Over the last twenty-five years research into the SBM process has been extensive, in an effort to improve preform design. This research has been focused on both experimental characterisation techniques to provide appropriate boundary conditions, and material properties. Based on this experimental characterisation, sophisticated simulations of the process have been developed, to reduce the economic cost of preform design.

Simulations of the SBM process have been outlined in the literature by several authors [16, 17, 18]. Typically, these utilise the finite element method to simulate the process. Yang et al. 18 simulated the SBM process using the commercial finite element package ABAQUS ${ }^{\circledR}$ Explicit [19].This study highlighted issues surrounding the use of the finite element method, as element distortion problems were encountered, which caused the numerical solution scheme to fail. Menary [20] came to a similar conclusion, suggesting that the large deformation ne- 
cessitated the use of shell elements. These issues provide a clear incentive to investigate this problem with a meshfree approach.

Cosson et al. 21 proposed the use of the constrained natural element method (C-NEM) 22 to simulate the stretch blow moulding. They found that the use of a meshfree method allows for the development of high strains without the need for remeshing, which can lead to possible solution degradation. Cosson et al. 23] furthered this study through the inclusion of a mould, and again applying the C-NEM method, they noted a similar benefit as before, with the meshfree method mitigating the need for re-meshing. The benefits outlined in this paper provide a strong argument for pursuing meshfree simulations of polymer forming processes, due to the high strains present in these processes, which can be problematic for the finite element method. However, validation of this simulation was limited to qualitative analysis and therefore the predictive capabilities of the meshfree simulations are untested.

The objective of this paper is to display the potential of meshfree methods as an alternative numerical technique to simulate material forming processes. Through simulations of the stretch blow moulding process, validated with experimental data outlined in this paper, we show that the meshfree simulation is highly capable in predicting the final shape of the bottle. This is particularly promising given that finite element simulations of this process require the use of shell elements, which are not valid at the base of the preform due to the thickness of the geometry. Due to the absence of a mesh, the meshfree simulation of this process does not require the same assumption. Therefore, experimental parameters, such as the through-thickness temperature profile have the potential to be investigated.

Firstly, a brief introduction to meshfree methods is presented, followed by the development of the numerical equations necessary for the simulation. The formulation developed in this work features nodal integration and two stabilisation schemes, which address stability issues faced by conventional nodal integration schemes. This results in a computationally efficient and numerically stable integration scheme. The meshfree formulation is validated against several finite deformation test problems.

\section{Element-free Galerkin Method}

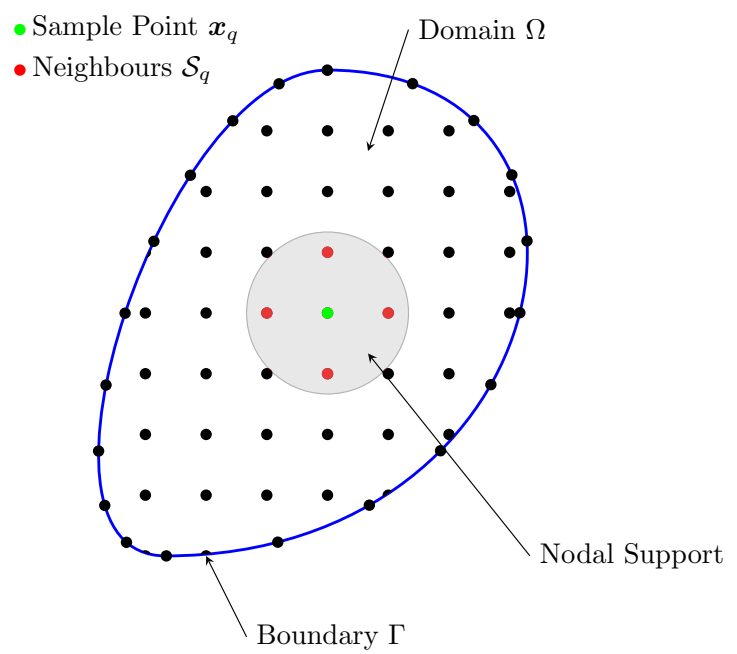

Figure 2: Meshfree discretization

In the element-free Galerkin method [24, the approximation of a scalar field $u(\boldsymbol{x})$ within the domain $\Omega \subset \mathbb{R}^{d}$ is constructed from a set of nodes $\left\{\boldsymbol{x}_{I}\right\}_{I=1}^{N}$ scattered over the domain, each with nodal value $u_{I}$, Figure 2. The value of the field variable at any 
point, $\boldsymbol{x}_{q}$, is obtained from a polynomial approximation,

$$
u\left(\boldsymbol{x}_{q}\right) \approx u^{h}\left(\boldsymbol{x}_{q}\right)=\boldsymbol{p}^{T}\left(\boldsymbol{x}_{q}\right) \boldsymbol{a}\left(\boldsymbol{x}_{q}\right),
$$

where $\boldsymbol{p}\left(\boldsymbol{x}_{q}\right)$ is a polynomial basis and $\boldsymbol{a}\left(\boldsymbol{x}_{q}\right)$ the coefficients of the approximation. The unknown coefficients are found from a moving least squares [25] fit of the data, which yields the least squares functional,

$$
J\left(\boldsymbol{x}_{q}\right)=\sum_{I}^{N p} w\left(\boldsymbol{x}_{q}-\boldsymbol{x}_{I}\right)\left[\boldsymbol{p}^{T}\left(\boldsymbol{x}_{I}\right) \boldsymbol{a}\left(\boldsymbol{x}_{q}\right)-u_{I}\right]^{2},
$$

where $w\left(\boldsymbol{x}_{q}-\boldsymbol{x}_{I}\right)$ is a weight function that assigns a domain of influence, $d_{I}$, to each node. In this work, the cubic spline weight function is used, defined by the piecewise equation

$$
w(r)= \begin{cases}\frac{2}{3}-4 r^{2}+4 r^{3}, & \text { if } r \leq \frac{1}{2} \\ \frac{4}{3}-4 r+4 r^{2}-\frac{4}{3} r^{3}, & \text { if } \frac{1}{2}<r \leq 1 \\ 0 & \text { if } r>1\end{cases}
$$

where $r$ is the normalised radius $r=\left|\boldsymbol{x}-\boldsymbol{x}_{I}\right| / d_{I}$. The shape function support size, $d_{I}$, is found by determining the minimum size $q_{I}$, such that three nodes are within each domain of influence and then scaling by a factor $d_{\max }$, i.e. $d_{I}=q_{I} \times d_{\max }$.

Minimisation of (2) yields the meshfree shape functions, $\phi_{I}(x)$, which are used to approximate the field variable through,

$$
u^{h}(x)=\sum_{I}^{N p} \phi_{I}(\boldsymbol{x}) u_{I}
$$

where $N_{p}$ is the number of nodes whose support contains the point $\boldsymbol{x}$. The full details of this minimisation procedure and construction of the shape functions can be found in Liu and $\mathrm{Gu}$ [26, Chapter $3]$.
Within this work a Lagrangian kernel is used, where the neighbours of a point are held constant throughout. This provides a significant computational benefit, as the shape functions are only obtained during the pre-processing stage. Additionally, Lagrangian kernels display increased stability in large deformation and avoid the numerical fracture that can occur with a Eulerian kernel [27].

\section{Lagrangian Meshfree Explicit Formula- tion}

In combination with the Lagrangian meshfree kernel, a Lagrangian virtual work formulation is used to generate the discrete system equations. Consider the equilibrium of a body initially occupying the region $\mathcal{B}_{0}$. Firstly, it is assumed that the boundary can be divided into a displacement boundary, $\Gamma_{u}$, where kinematic constraints are prescribed, and a traction boundary $\Gamma_{t}$, where prescribed loading is applied. Denote $\Omega^{0}$ the set of material coordinates, $X$, which define the body at $t=0$, along with the material boundaries $\Gamma_{t}^{0}$ and $\Gamma_{u}^{0}$. In each time instance $t \in[0, T]$, the equation of motion for the body is given by

$$
\nabla_{0} \cdot \boldsymbol{P}=\rho_{0} \boldsymbol{a}, \boldsymbol{X} \in \Omega_{0}, \forall t \in[0, T]
$$

with boundary conditions,

$$
\begin{array}{r}
\boldsymbol{P N}=\boldsymbol{T} \text { for all } \boldsymbol{X} \in \Gamma_{t}^{0}, \\
\boldsymbol{u}=\overline{\boldsymbol{u}} \text { for all } \boldsymbol{X} \in \Gamma_{u}^{0},
\end{array}
$$

where $\boldsymbol{P}$ is the first Piola-Kirchhoff stress, $\rho$ the density, $\nabla_{0}$ the gradient operator in the material coordinate $\boldsymbol{X}, \boldsymbol{N}$ a unit normal on the surface $\Gamma_{t}$, and $\boldsymbol{a}$ the material acceleration. Multiplying through 
(5) by a set of virtual displacements, $\delta \boldsymbol{u}$, yields

$$
\left(\nabla_{0} \cdot \boldsymbol{P}-\rho \boldsymbol{a}\right) \cdot \delta \boldsymbol{u}=0
$$

Integrating (8), and applying the divergence theorem, yields the well known principle of virtual work, given in terms of the material coordinates as

$$
\int_{\Omega^{0}}\left[\rho_{0} \delta \boldsymbol{u}_{i} \boldsymbol{a}_{j}+\delta \boldsymbol{F}_{i j} \boldsymbol{P}_{i j}\right] d \Omega-\int_{\Gamma_{t}^{0}} \delta \boldsymbol{u} \cdot \boldsymbol{T} d \Gamma=0
$$

where $\delta \boldsymbol{F}$ is the variation of the deformation gradient. Substituting the MLS approximation (4), into (9), yields the semi-discrete equations

$$
m_{I} \boldsymbol{a}_{I}=\boldsymbol{f}_{I}^{e x t}-\boldsymbol{f}_{I}^{i n t}
$$

where $m_{I}$ is the mass of node $I$. The internal and external force vectors are given by

$$
\begin{array}{r}
\boldsymbol{f}_{I}^{e x t}=\int_{\Gamma_{t}^{0}} \phi_{I} \boldsymbol{T} d \Gamma, \\
\boldsymbol{f}_{I}^{i n t}=\int_{\Omega^{0}} \boldsymbol{B}_{I}^{T} \hat{\boldsymbol{P}} d \Omega .
\end{array}
$$

In the equations above, $\hat{\boldsymbol{P}}$ is the Voigt form of the first Piola-Kirchhoff stress tensor, and $\boldsymbol{B}_{I}$ the strain-displacement matrix. The nodal mass is found from mass lumping via the row-sum technique, which yields,

$$
m_{I}=\sum_{K=1}^{N} \int_{\Omega_{0}} \rho^{0} \phi_{I} \phi_{K} d \Omega=\int_{\Omega^{0}} \rho^{0} \phi_{I} d \Omega .
$$

The integrals in 11 - 13 are evaluated using nodal integration, which assigns a volume to each node, yielding the integral approximation

$$
\int_{\Omega_{0}}(\cdot)=\sum_{k}^{N}(\cdot)_{k} v_{k}
$$

where $v_{k}$ is a volume assigned to each node. For boundary terms the quadrature scheme is given in

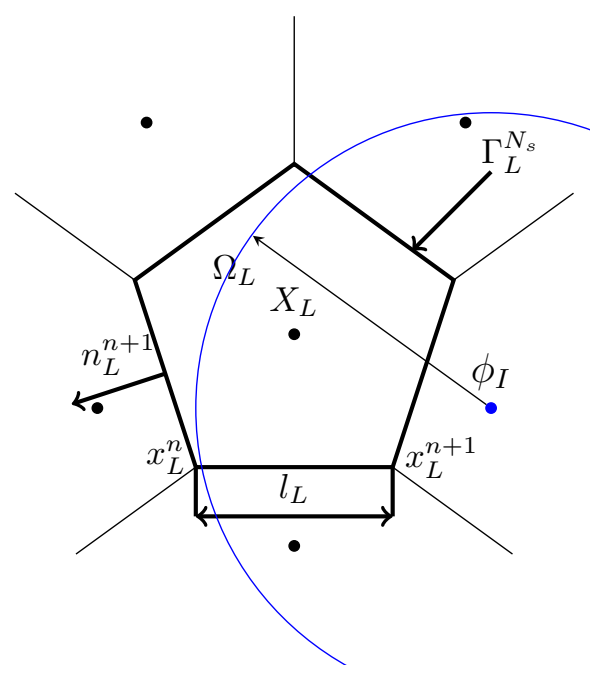

Figure 3: Typical Voronoi cell used for nodal integration

terms of the boundary area, $\omega_{k}$, as

$$
\int_{\Gamma_{0}}(\cdot)=\sum_{k}^{N}(\cdot)_{k} \omega_{k} .
$$

Evaluating 11 - 13 using the integral approximations 14 and 15, yields the following nodal force, and mass terms:

$$
\begin{aligned}
\boldsymbol{f}_{I}^{e x t} & =\sum_{k=1}^{N_{b}} \phi_{I} \boldsymbol{T} \omega_{k}, \\
\boldsymbol{f}_{I}^{i n t} & =\sum_{k=1}^{N_{n}} \boldsymbol{B}_{I}^{T} \boldsymbol{P} v_{k}, \\
m_{I} & =\sum_{k=1}^{N_{n}} \rho_{0} \phi_{I}\left(x_{k}\right) v_{k} .
\end{aligned}
$$

In this work, the nodal volumes are based on a Voronoi diagram, Figure 3. With the use of a Voronoi diagram, the stabilised conforming nodal integration (SCNI) method proposed by Chen et al. 28] can be used. This integration scheme is constructed to satisfy the divergencefree condition necessary for first-order convergence. The divergence-free condition is satisfied through 
a smoothed deformation gradient, which is constructed using the Voronoi cell surrounding each of the nodes. This approach leads to a smoothed deformation gradient of the form,

$$
\boldsymbol{F}_{i j}\left(\boldsymbol{X}_{L}\right)=\frac{1}{A_{L}} \int_{\Gamma_{L}} \phi_{I} u_{I i} N_{j} d \Gamma+\delta_{i j} .
$$

Evaluation of the integral 19 leads to a smoothed displacement gradient matrix, $\overline{\boldsymbol{B}}$, which for two dimensions is given by

$$
\overline{\boldsymbol{B}}_{I}\left(X_{L}\right)=\left[\begin{array}{cc}
b_{1 I} & 0 \\
0 & b_{2 I} \\
b_{2 I} & 0 \\
0 & b_{1 I}
\end{array}\right]
$$

where,

$$
b_{j I}=\frac{1}{A_{L}^{x}} \int_{\Gamma_{L}^{X}}\left(\phi_{I} N_{j}\right) d \Gamma .
$$

The components of the smoothed straindisplacement relationship 20 are integrated numerically. In this work a two point trapezoidal scheme is used, which leads to the following recursive relationship for the components, $b_{i I}$,

$$
b_{i I}=\sum_{n=1}^{N s}\left[\frac{1}{2}\left(N_{i L}^{n} l_{L}^{n}+N_{i L}^{n+1} l_{L}^{n+1}\right) \phi_{I}\left(x_{L}^{n+1}\right)\right],
$$

where $l_{L}^{n}$ and $n_{L}^{n}$ are the length, and normal vector of the $n t h$ segment of the cell that surrounds $X_{L}$,

\section{Figure 3 .}

The derivations above hold for 2D plane problems. To develop an axisymmetric meshfree formulation we consider a body described by the material cylindrical coordinates $(R, \theta, Z)$, Figure 4 . In this figure, $d L(\xi)$ refers to a parametric representation of the surface length, with $d L=d Z$ holding for planar surfaces. Using this cylindrical system, the material differential volume $\left(d \Omega^{0}\right)$ and surface

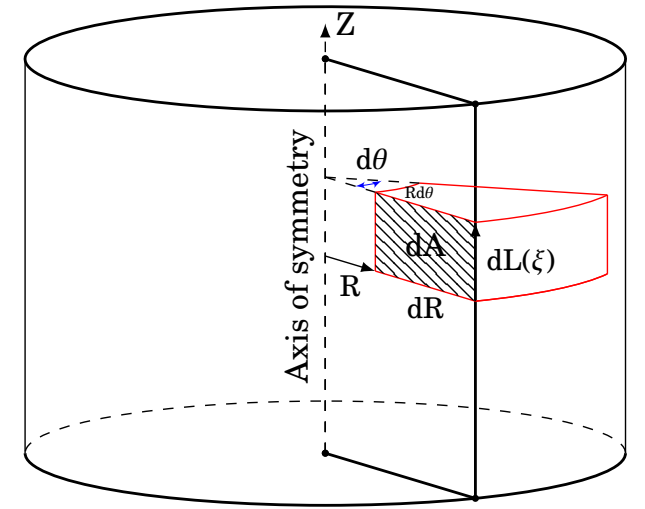

Figure 4: Axisymmetric element in the material cylindrical coordinates $(R, Z, \theta)$.

$\left(d \Gamma^{0}\right)$ elements are given by,

$$
\begin{aligned}
& d \Omega^{0}=R d \theta d A, \\
& d \Gamma^{0}=R d \theta d L,
\end{aligned}
$$

which follow directly from Figure 4. Using the relations 23 , the virtual work expression $(9)$ is rewritten as,

$$
\begin{gathered}
\int_{0}^{2 \pi} \int_{A}\left[\rho_{0} \delta \boldsymbol{u}_{i} \boldsymbol{a}_{j}+\delta \boldsymbol{F}_{i j} \boldsymbol{P}_{i j}\right] R d A d \theta \\
-\int_{0}^{2 \pi} \int_{L} \delta \boldsymbol{u} \cdot \boldsymbol{T} R d L d \theta=0
\end{gathered}
$$

which may be rewritten as,

$$
\begin{gathered}
2 \pi\left(\int_{A}\left[\rho_{0} \delta \boldsymbol{u}_{i} \boldsymbol{a}_{j}+\delta \boldsymbol{F}_{i j} \boldsymbol{P}_{i j}\right] R d A\right. \\
\left.-\int_{L} \delta \boldsymbol{u} \cdot \boldsymbol{T} R d L\right)=0 .
\end{gathered}
$$

The axisymmetric weak form 25) has the same structure as (9) and therefore the equation of motions in terms of the external and internal forces (10) holds. Due to the symmetry of the problem, there are two degrees of freedom, such that the displacement and traction degrees of freedom in 25 
are given by,

$$
\begin{gathered}
\boldsymbol{u}=\left[u_{R}, u_{Z}\right]^{T} \\
\boldsymbol{T}=\left[T_{R}, T_{Z}\right]^{T} .
\end{gathered}
$$

The deformation gradient for the axisymmetric formulation has an additional row due to the hoop component $F_{\theta \theta}$,

$$
\boldsymbol{F}=\left[\begin{array}{ccc}
\frac{\partial u_{R}}{\partial R} & \frac{\partial u_{R}}{\partial Z} & 0 \\
\frac{\partial u_{Z}}{\partial R} & \frac{\partial u_{Z}}{\partial Z} & \\
0 & 0 & \frac{u_{R}}{R}
\end{array}\right] .
$$

With regards to the smoothed deformation gradient outlined in 197, smoothing of this hoop component is not performed. Instead, the hoop component, $F_{\theta \theta}$ is evaluated nodally as in 29. This leads to the following axisymmetric strain displacement matrix,

$$
\overline{\boldsymbol{B}}_{I}\left(X_{L}\right)^{a x i}=\left[\begin{array}{cc}
b_{1 I} & 0 \\
0 & b_{2 I} \\
b_{2 I} & 0 \\
0 & b_{1 I} \\
\frac{\phi_{I}}{R\left(X_{L}\right)} & 0
\end{array}\right] .
$$

The equations of motion (10) are integrated in time using an explicit central difference timestepping scheme [30], with half time-step velocities, which yields the kinematic update formulas,

$$
\begin{aligned}
t_{n+1} & =t_{n}+\Delta t, \\
\boldsymbol{v}_{n+\frac{1}{2}} & =\boldsymbol{v}_{n-\frac{1}{2}}+\Delta t \boldsymbol{a}_{n}, \\
\boldsymbol{u}_{n+1} & =\boldsymbol{u}_{n}+\Delta t \boldsymbol{v}_{n+\frac{1}{2}} .
\end{aligned}
$$

As this is an explicit method, it is conditionally stable, with a critical timestep based on the maximum natural frequency of the system:

$$
\Delta t_{c}=\frac{2}{\omega_{\max }} .
$$

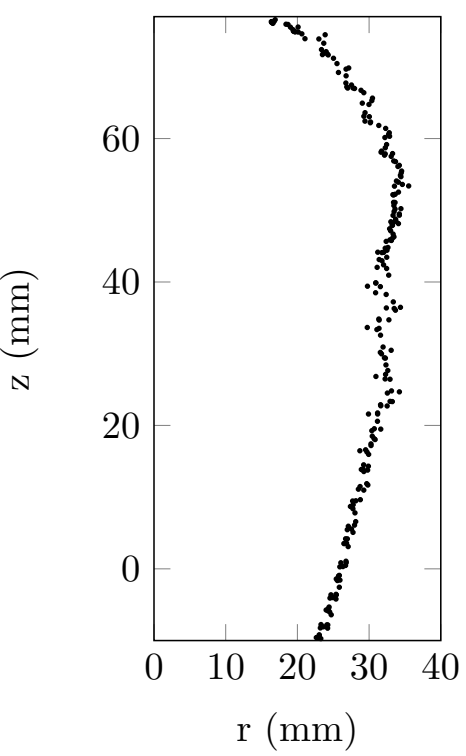

Figure 5: Unstable response, leading to serve particle disorder

An upper bound of the maximum natural frequency, $\omega_{u b}$, for nodally integrated meshfree methods, was proposed by [31 as,

$$
\omega_{u b}^{2}=(\lambda+2 \mu) \max _{I=1, N}\left(\max _{i=1,3} \sum_{J} \boldsymbol{B}_{i J}^{I} \boldsymbol{B}_{i J}^{I}\right),
$$

where $\lambda$ and $\mu$ are the Lamé parameters.

\subsection{Stress Point Integration}

Although the stabilised nodal integrations scheme used in this work does not suffer from any zero-energy modes, as typical of one-point finite element integration schemes, it suffers from the onset of spurious modes of deformation [31, which have the potential to be excited when the discretization is fine. Evidence of this can be seen in Figure 5 , which is a result that arose during one of the stretch blow moulding simulations, which are presented towards the end of this paper. To avoid this phenomenon we propose the use of stress points, which have been used frequently to stabilise particle-based 


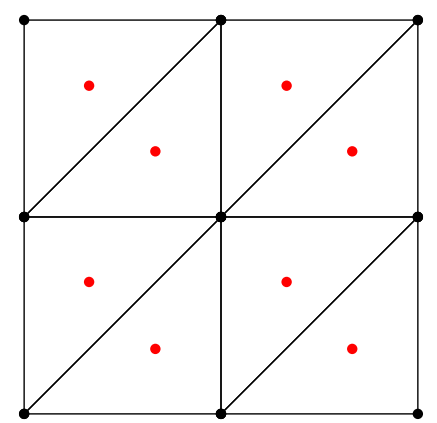

Figure 6: Addition of stress points to the original triangulation

simulations 32. These stress points are introduced at the barycentres of the Delaunay triangulation, Figure 6 . With the addition of these stress points, the body is discretised by a set of $N_{n}$ nodes and $N_{s p}$ stress points, such that volume of the body, $V$, is given by,

$$
\sum_{K}^{N_{n}} v_{k}+\sum_{K}^{N_{s p}} v_{k}=V .
$$

The volume associated to the stress and nodal points is found by constructing a Voronoi diagram of the complete point-set $N_{n}$ and $N_{s p}$. This ensure that (32) is satisfied. The displacement at these stress points is determined from the surrounding nodes, therefore only the stress, $\boldsymbol{P}^{s p}$, needs to be evaluated at these points [11. The displacement at these stress points follows from (4) as,

$$
u\left(\boldsymbol{x}_{s p}\right)=\sum_{I}^{N_{p}} \phi_{I}\left(\boldsymbol{x}_{s p}\right) u_{I},
$$

where $\phi_{I}\left(x_{s p}\right)$ is the value of the shape function of node $x_{I}$ at the stress point $x_{s p}$. With the use of stress points, the internal forces are given by,

$$
\begin{aligned}
f_{I}^{i n t}= & \sum_{k \in N_{N}^{I}} \boldsymbol{B}_{I}\left(x_{k}\right)^{T} \boldsymbol{P}^{n} v_{k} \\
& +\sum_{k \in N_{s p}^{I}} \boldsymbol{B}_{I}\left(x_{k}\right)^{T} \boldsymbol{P}^{s p} v_{k},
\end{aligned}
$$

where the sets $N_{N}^{I}$ and $N_{s p}^{I}$ are the nodes and stress points which contribute to the internal forces at the node $\boldsymbol{x}_{I}$.

\subsection{Boundary Conditions}

As the moving least squares shape functions are not interpolants, imposing essential boundary conditions is more challenging than in the finite element method. To implement boundary conditions within an explicit meshfree scheme, a predictorcorrector type approach, as similar to the finite element method, is typically followed 33, 34. Within a meshfree method, the predictor-corrector scheme differs from the finite element implementation due to the influence of internal nodes on the boundary displacements.

In this work, the method proposed by Joldes 34 . is used to apply boundary conditions. Within this method, the authors developed a method suitable for explicit schemes, where an additional force is included in the external and internal force balance (10). This added force is determined based on requiring that kinematic boundary conditions are satisfied at each time step. The force is determined by considering a predictor-corrector kinematic update, defined by

$$
\boldsymbol{u}=\boldsymbol{u}^{\text {pre }}+\boldsymbol{u}^{\text {corr }},
$$

Generating the displacement correction requires the consideration of an additional force arising from the boundary conditions. In the element-free Galerkin method, due to the use of non-interpolating shape functions, an additional force, $\boldsymbol{F}^{\text {ess }}$, exists on the displacement boundaries, given by

$$
\boldsymbol{F}_{I}^{e s s}=\int_{\Gamma_{u}} \phi_{I} \boldsymbol{T} d \Gamma
$$


where $\mathrm{T}$ is the surface traction required to apply the boundary conditions. Discrete evaluation of 36 can be considered by distributing $n_{k}$ nodes on the essential boundary, which leads to the following matrix form:

$$
\boldsymbol{F}^{e s s}=\boldsymbol{V} \boldsymbol{T},
$$

where,

$$
\boldsymbol{V}_{J k}=\phi_{J}\left(\boldsymbol{n}_{k}\right) .
$$

Within a meshfree method, the displacement $\boldsymbol{u}$ is typically referred to as the generalised displacement, which is related to the nodal (actual) components, $\boldsymbol{d}$, of the displacement through the transformation

$$
d=\Lambda u
$$

where $\Lambda$ is the transformation matrix defined by,

$$
\Lambda_{I J}=\phi_{I}\left(\boldsymbol{X}_{J}\right) .
$$

On the essential boundaries this transformation should yield the prescribed displacement field $\overline{\boldsymbol{u}}$ :

$$
\overline{\boldsymbol{u}}=\boldsymbol{\Lambda}^{e s s} \boldsymbol{u}
$$

with,

$$
\boldsymbol{\Lambda}^{e s s}=\phi_{I}\left(\boldsymbol{X}_{J}\right) \forall J \text { on } \Gamma_{u} .
$$

Application of a central difference time-stepping scheme, along with the definition of the essential boundary force above, yields the following predictor-corrector formula,

$$
\boldsymbol{u}^{\text {corr }}=\boldsymbol{P}\left(\overline{\boldsymbol{u}}-\boldsymbol{\Lambda}^{\text {ess }} \boldsymbol{u}\right),
$$

which combined with (35) yields the update formula for the displacement. The matrix $\boldsymbol{P}$ in (43) arises due to the application of the central time-stepping scheme and is given by

$$
P=\boldsymbol{M}^{-1} \boldsymbol{V}\left(\boldsymbol{\Lambda}^{e s s} \boldsymbol{M}^{-1} \boldsymbol{V}\right)^{-1} .
$$

As a Lagrangian formulation is used, the matrices in (44) are constant and are found in the preprocessing stage, providing a significant computational saving.

\section{Numerical Validation}

To validate the proposed algorithm we consider two hyperelastic problems. The structure of the programme to solve these problems is shown in Appendix A. The benefit of meshfree schemes based on SCNI is the reduced sensitivity to irregular nodal distributions, which has also been reported in [35, 29]. This is a particularly attractive property for material forming, where complex geometries can inhibit the ability to generate high-quality uniform discretisations.

\subsection{Tip-loaded Beam}

The first problem is a tip loaded beam, which is shown in Figure 7. The stress-strain behaviour of the beam is characterised by the St-Venant Kirchoff material model, where the first Piola-Kirchhoff stress, $\boldsymbol{P}$, is described in terms of the Lame parameters $\mu$ and $\lambda$ as,

$$
\boldsymbol{P}=\boldsymbol{F}(\lambda(\operatorname{tr} \boldsymbol{E}) \boldsymbol{I}+2 \mu \boldsymbol{E}),
$$

and $\boldsymbol{E}$ is the Green-Lagrange strain tensor, given by:

$$
\boldsymbol{E}=\frac{1}{2}\left(\boldsymbol{F}^{T} \boldsymbol{F}-\mathbf{I}\right) .
$$

The normalised tip displacement $(w / L)$ is described by the elliptical equation 36

$$
\frac{w}{L}=1-\sqrt{\frac{4 E I}{P L^{2}}}[E(k)-E(k, \phi)],
$$


where $I$ is the moment of inertia of the beam and $E(k)$ and $E(k, \phi)$ are elliptical integrals defined by:

$$
E(k)=\int_{0}^{\pi / 2} \sqrt{\left(1-k^{2} \sin ^{2} t\right) d t}
$$

and,

$$
E(k, \phi)=\int_{0}^{\phi} \sqrt{\left(1-k^{2} \sin ^{2} t\right) d t} .
$$

To obtain a solution to this problem a numerical approach has to be adopted, which is described in detail in Timoshenko and Gere [37, Chapter 7], and as such will not be repeated here. Instead, we make use of the solution provided by Mattiasson [38.

For Gauss integration, we consider a second-order scheme, which corresponds to three integration points per triangle. The domain sizes are found by ensuring that at least three nodes are within the domain. In this example we consider the variation in the solution against the domain scaling factor $d_{\max }$ and the nodal density. The reason for investigating the support size parameter is that in explicit meshfree methods it has been shown that large domains of influence, coupled with the row-sum techniques 13 leads to inertia errors 31.

\subsubsection{Results}

The comparison between the end displacement for the reference solution and the meshfree solution are given in Figure 8 and Figure 9, for varying nodal densities. In the following figures, NI refers to nodal integration using the stabilised conforming nodal integration approach, and GI to a secondorder Gauss-type scheme. The errors stated in this section are obtained using an $\mathrm{R}$ squared $\left(R^{2}\right)$ value, where $R^{2}=1$ refers to $0 \%$ error.
Lower Density. In Figure 8 the solution obtained for a low number of nodes is shown. The application of Gauss integration in this scenario leads to very poor results, which is expected due to the nodal arrangement, which leads to a strong misalignment between the shape function support, and the integration points, an issue that was highlighted by Dolbow and Belytschko [39]. These results highlight the effect that the support size has on the accuracy of the numerical integration, which is particularly prevalent for disordered nodal arrangements. This influence is confirmed by the differences in the solution observed for an increase in the support size parameter $d_{\max }$, which in this case leads to an improvement in the GI solution. This behaviour is far less prominent in nodal integration, as evidenced by the almost identical results produced for $d_{\max }=1.5$, and $d_{\max }=2.5$. We suspect that these differences occur in the construction of the internal forces vector, which is assembled at each of the quadrature points. At each of these sampling points, the largest contribution to the local internal force vector is typically from the nodes closest to the sampling point. For a Gauss integration scheme, with a non-uniform nodal distribution, the effective closeness of the nodes to the quadrature point is dependent on the domain size, which leads to a local internal force vector which is sensitive to the domain size. In nodal integration, this sampling point coincides with the node, and therefore the (large) contribution to the internal force vector from the closest node is always accounted for regardless of the domain size, which leads to the reduced sensitivity. 


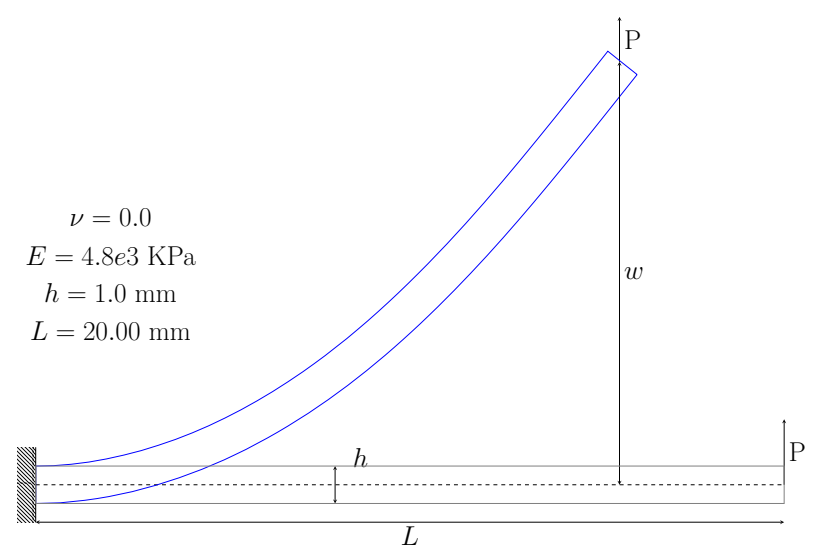

Figure 7: Tip-loaded cantilever beam geometry

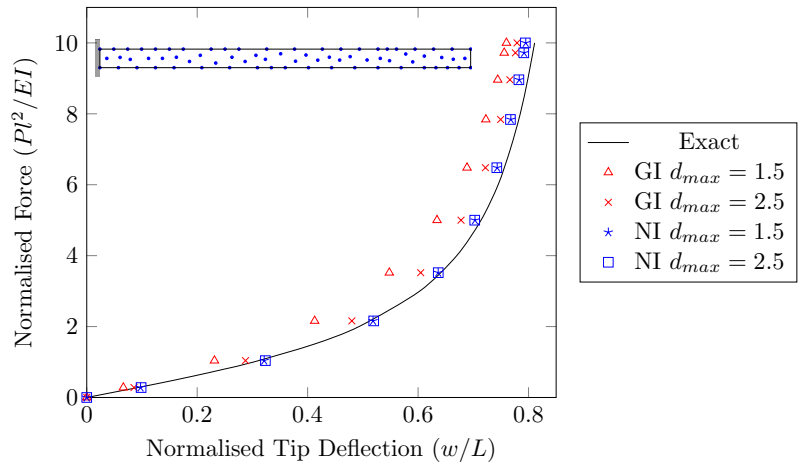

Figure 8: Tip-loaded cantilever beam: comparison between exact and numerical solution (57 nodes)

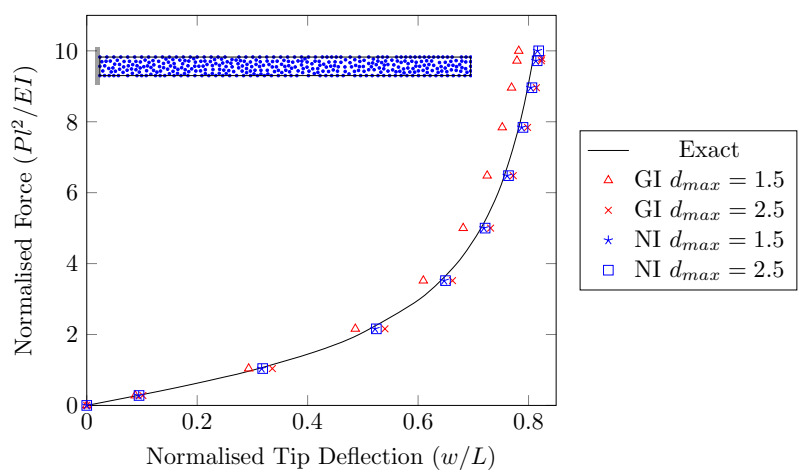

Figure 9: Tip-loaded cantilever beam: comparison between exact and numerical solution (206 nodes)

Increasing nodal density. To increase the accuracy to within a $5 \%$ tolerance, a significant increase in the number of nodes was required, as shown in Figure 9 , where the error for $d_{\max }=2.5$ is now $4 \%$. However, as illustrated in this figure the error for small domains of influence remains high at $12 \%$. In the nodal integration results for this increased discretisation, the error is less than $1 \%$ in both cases. These results highlight the ability of SCNI to provide accurate results in the presence of a small number of nodes, and tight support domains. These features are very attractive as it should lead to a lower computational cost, a feature which is now investigated. To verify that convergence has been achieved, the solution obtained from 380 nodes, using stabilised nodal integration, is shown in Figure 10. As seen in this figure, the numerical solution remains in a good agreement with the exact solution, signifying that convergence has been attained. The error attributed to each nodal distribution is shown in Table 1

Computational Cost. The computational cost for SCNI using an implicit meshfree method was in- 


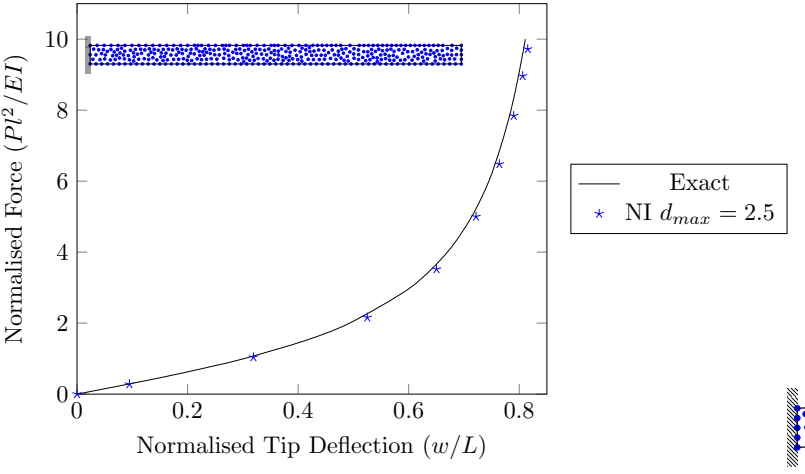

(a)

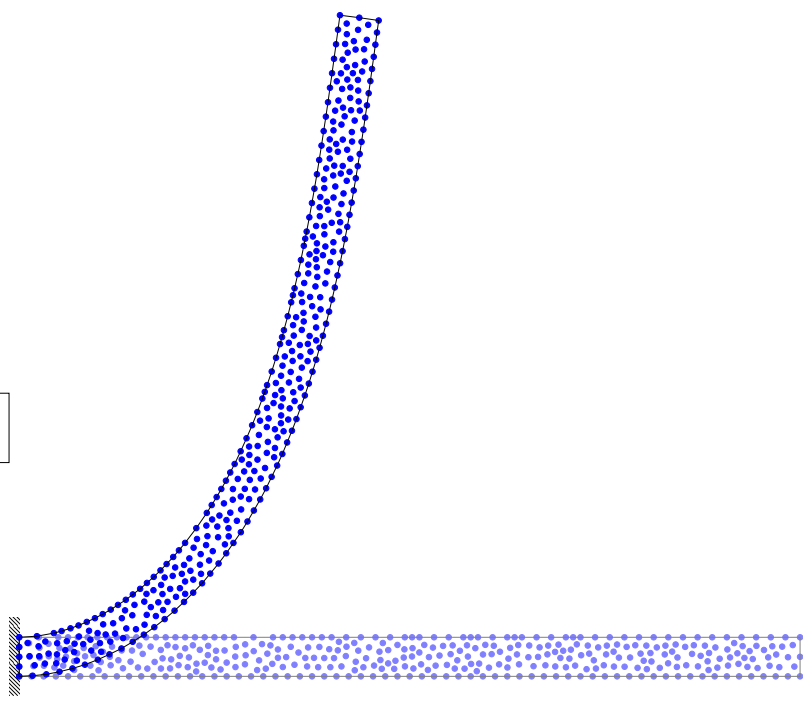

(b)

Figure 10: Tip-loaded cantilever beam (380 nodes): (a) final tip displacement solution and (b) final displacement

Table 1: Tip-loaded cantilever beam: $R^{2}$ value for varying nodal distribution using nodal integration

\begin{tabular}{ccc}
\hline \multirow{2}{*}{ Nodes } & \multicolumn{2}{c}{ Nodal integration $-R^{2}$} \\
\cline { 2 - 3 } & $d_{\max }=1.5$ & $d_{\max }=2.5$ \\
\hline 57 & 0.96 & 0.98 \\
109 & 0.97 & 0.98 \\
206 & 0.99 & 0.99 \\
380 & 0.99 & 0.99 \\
\hline
\end{tabular}

vestigated by [28], where a significant improvement was observed. In this work, we investigate the cost savings within the explicit element-free Galerkin method, which is expected to be high as the cost of an explicit method is related to the construction of the internal force vector, which is assembled at each of the quadrature points. For this analysis, the computational cost is determined based on the running time of the explicit timing step- ping routine and does not include any pre- or postprocessing tasks, such as shape function determination and neighbour searches. The computational cost is normalised against a 57 -node SCNI scheme and is shown in Figure 11, where GI refers to a second-order Gauss scheme. As illustrated in this figure, the cost of Gauss integration is significantly larger than the SCNI scheme, and without any benefits in terms of accuracy.

\subsection{Hyperelastic pressure-lodead cylinder}

The second problem we investigate is that of a pressure-loaded cylinder, shown in Figure 12. This problem was investigated by Rivlin [40, where the solution for the internal pressure is given as

$$
\begin{aligned}
P\left(r_{1}\right)= & \left(C_{1}+C_{2}\right)\left[\frac{\left(r_{1}^{2}-R_{2}^{2}\right)\left(R_{1}^{2}-R_{1}^{2}\right)}{r_{2}^{2}\left(r_{2}^{2}+R_{1}^{2}-R_{2}^{2}\right)}\right. \\
& \left.+\ln \left(\frac{r_{2}^{2}}{r_{2}^{2}+R_{1}^{2}-R_{2}^{2}}\right)+\ln \frac{R_{1}^{2}}{R_{2}^{2}}\right],
\end{aligned}
$$




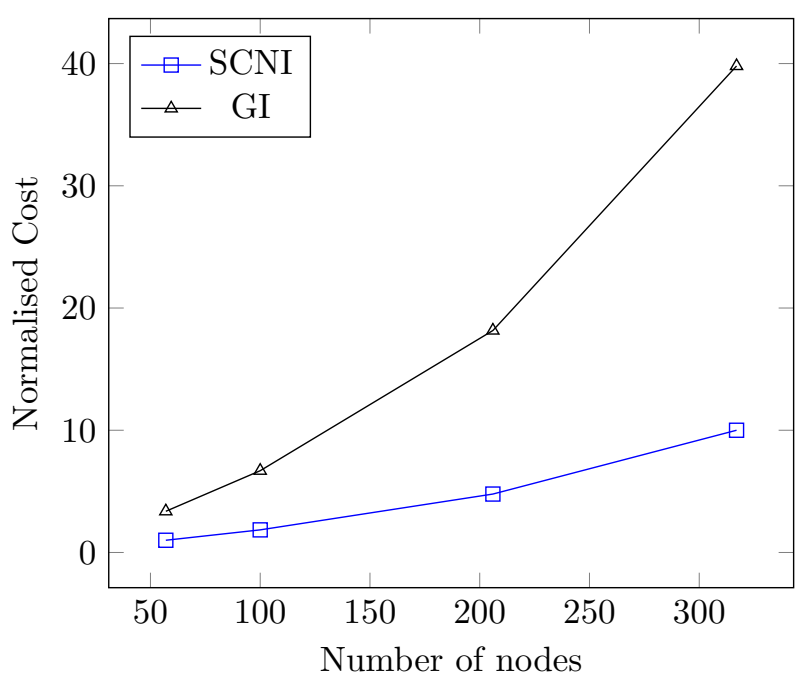

Figure 11: Tip-loaded cantilever beam: Computational cost comparison

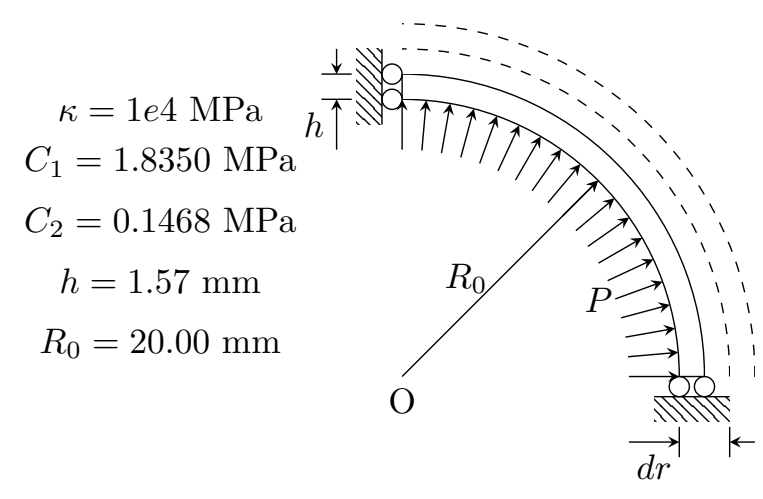

Figure 12: Mooney-Rivlin Cylinder (quarter model)

where $r_{1}$ and $r_{2}$ are the current internal, and external radii of the cylinder, and $R_{1}$ and $R_{2}$ the initial internal and external radii respectively. The constants $C_{1}$ and $C_{2}$ are those of the Mooney-Rivlin type material, for which the strain energy is given by 41

$$
\psi=C_{1}\left(I_{1}-3\right)+C_{2}\left(I_{2}-3\right)+\frac{\kappa}{2}\left(\ln \left(I_{3}\right)\right)^{2} .
$$

The first Piola-Kirchhoff stress, $\boldsymbol{P}$, required for the solution procedure, can be determined from the strain energy density function (51) as,

$$
\begin{array}{r}
\boldsymbol{P}_{k j}=2 \boldsymbol{F}_{k i}\left[C_{1} \boldsymbol{\delta}_{i j}+C_{2}\left(\boldsymbol{C}_{n n} \boldsymbol{\delta}_{i j}-\boldsymbol{C}_{i j}\right)\right]- \\
2 \boldsymbol{F}_{k i}\left(C_{1}+2 C_{2}-\kappa \ln I_{3}\right) \boldsymbol{C}_{i j}^{-1} .
\end{array}
$$

The constants $\left\{C_{1}, C_{2}, \kappa\right\}$ used within this model are shown in Figure 12, where the high ratio of $\kappa$ to $C_{i}$ invokes the desired incompressibility. We consider a fixed discretization of 100 nodes, and once again apply SCNI and Gauss quadrature to perform numerical integration. For this analysis, a constant support size scaling parameter of $d_{\max }=2$ is used.

Pressure-displacement solution. As the first solution to this problem, we consider a second-order Gauss scheme and compare the results of the pressure displacement curve with the analytical solution in Figure 13. In this figure, an overly stiff behaviour for the second-order scheme (2-GI) can be seen, which is likely to be due to the high number of integration points, combined with a large bulk modulus. To avoid this stiff behaviour - which is not present in the SCNI solution - we consider using a lower order integration rule (1-GI). This is a common technique within the finite element method. Repeating this solution with a lower order of integration provides a good agreement between the analytical and meshfree solution, which in this case matches the accuracy of SCNI.

Despite the apparent accuracy of this solution, low order integration schemes, combined with meshfree methods, can lead to instabilities due to under-integration 24]. Within a dynamic timestepping procedure, errors due to under-integration can manifest themselves as spurious non-physical modes, which can lead to large errors in energy 


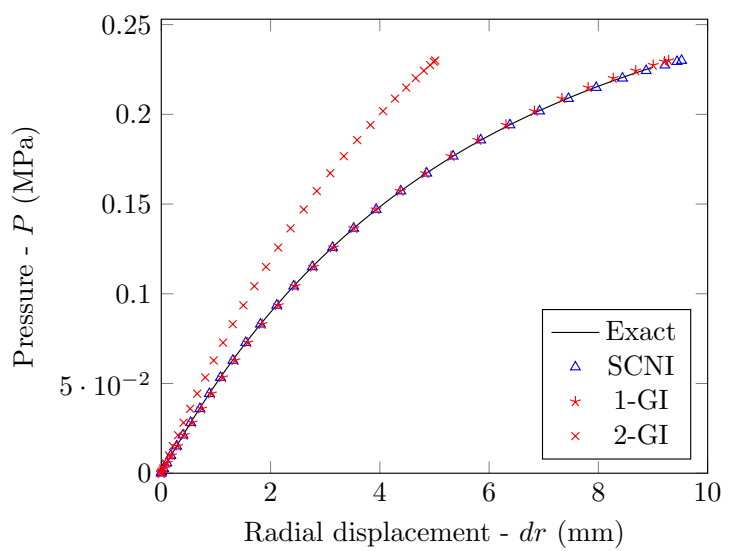

(a)

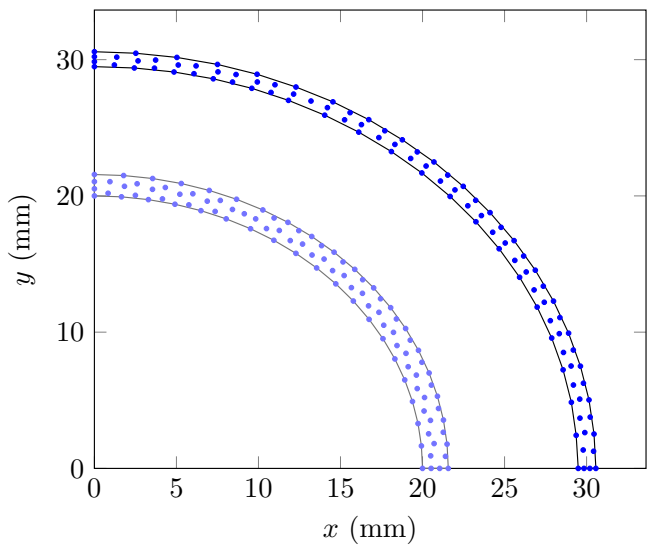

(b)

Figure 13: Mooney-Rivlin Cylinder: (a) comparison with the exact solution, and (b) displacement profile for SCNI

measurements [1]. Although SCNI and (1-GI) are both inherently one-point quadrature schemes, the former can pass a linear patch test 42, which ensures that convergence to the exact solution can be obtained with decreasing nodal spacing $h$. In contrast, a meshfree scheme integrated by one point Gauss quadrature cannot pass a linear patch test 43, therefore with decreasing nodal spacing, a solution will be reached that does not necessarily correspond to the exact solution. To examine whether this leads to undesired effects, the stress response is investigated.

Stress response. The stress response with one point Gauss integration and SCNI are shown in Figure 14 In both cases, the stress is plotted at the quadrature points, which for SCNI corresponds to the nodal location. The stresses are displayed in a Cartesian coordinate system $\{x, y\}$, such that $\sigma_{11}$ refers to $\sigma_{x x}$

As illustrated in this figure, the results for SCNI show that despite the coarse nodal distribution used, a smooth symmetrical variation in stress is observed. Conversely, in the Gauss integrated predictions, an oscillatory response is encountered, where it can be seen that at several different locations the stress component is equal to zero.

These observations highlight the difficulty of using one-point Gauss integration, combined with an irregular nodal distribution, within a standard meshfree formulation. These same difficulties are not faced by SCNI, where improved accuracy, reduced cost, and higher stability were seen throughout the numerical examples. These results highlight the benefits of using a nodally integrated meshfree method.

\section{Experimental Characterisation of SBM}

A series of experimental trials were conducted based on the free blow process, which corresponds to the stretch blow moulding process with the mould removed.

Experimental results were obtained using INDICATE [4], a novel instrumentation capable of cap- 


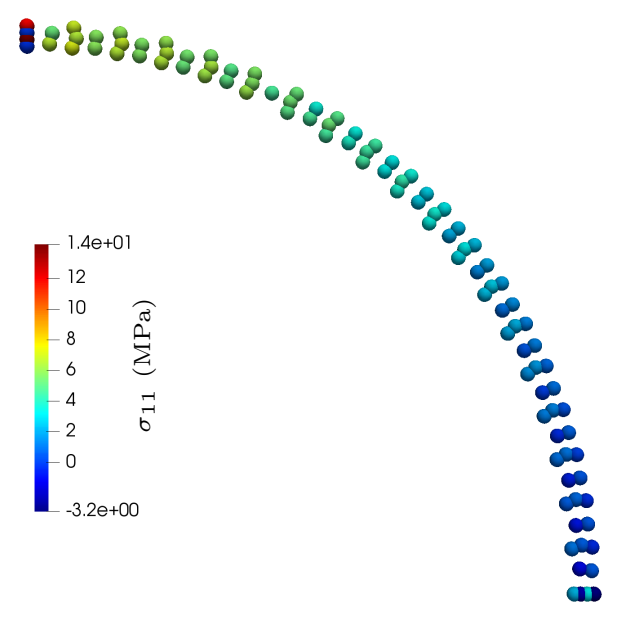

(a)

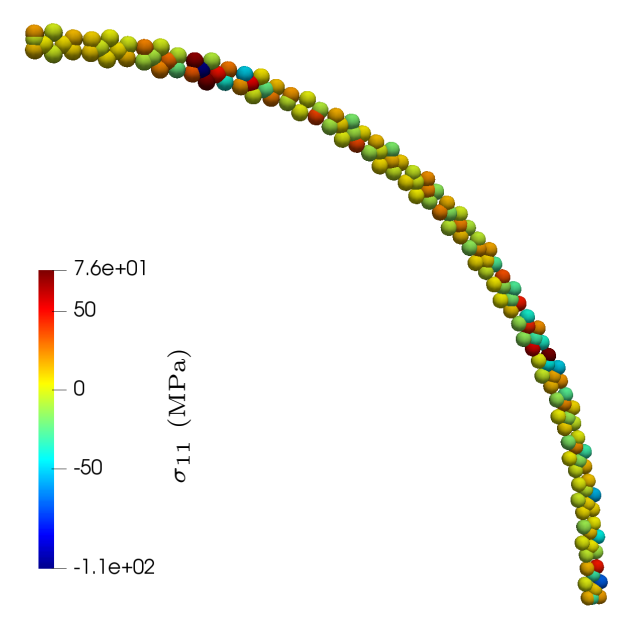

(b)

Figure 14: Mooney Rivlin Cylinder: Stress $\sigma_{11}$ for (a) SCNI and (b) 1-GI

turing the displacement, and strain evolution of the bottle using high-speed imaging. A full description of the experimental procedures can be found in [45, 46]. A $27 \mathrm{~g}$ preform was used for these trials, which is shown in Figure 15. The preforms were heated to $105^{\circ} \mathrm{C}$ using oil bath heating for $210 \mathrm{~s}$, to ensure that temperature equilibrium was reached. The mass flow rate of air into the cavity was determined by a line of pressure, which was held constant at 8 Bar, and the effective flow area, which was varied through adjustment of the flow restriction area. This flow rate was described by a dimensionless parameter $N(\cdot)$, which for this analysis was set at N2, N5 and N8. These flow rates were chosen so that a range of strain rates were applied to the preform. The stretch rod speed was set at $1 \mathrm{~m} / \mathrm{s}$ with a maximum travel of $100 \mathrm{~mm}$.

The strain and displacement histories were investigated at three points along the preform sidewall at $z=-26 \mathrm{~mm}, z=-46 \mathrm{~mm}$ and $z=-64 \mathrm{~mm}$,
Figure 15, using digital image correlation (DIC). In this figure, $z=0$ refers to the bottom of the neck support ring. The DIC technique tracks the evolution of a pattern, applied to the preform, with time. Through this evolution, the Lagrangian strain tensors can be defined, allowing for the strain field to be determined. This technique has frequently been applied to characterise the large strain behaviour of polymers [47, 46].

In the analysis which follows, the threedimensional strain map obtained from the DIC technique was reduced to an equivalent axisymmetric representation using the techniques outlined in [45. Using this method, the deformation at each sampling point is described by four axisymmetric true strain components: (1) hoop $\epsilon_{\theta \theta}$, (2) radial $\epsilon_{r r},(3)$ axial $\epsilon_{z z}$ and (4) shear $\epsilon_{r \theta}$. 


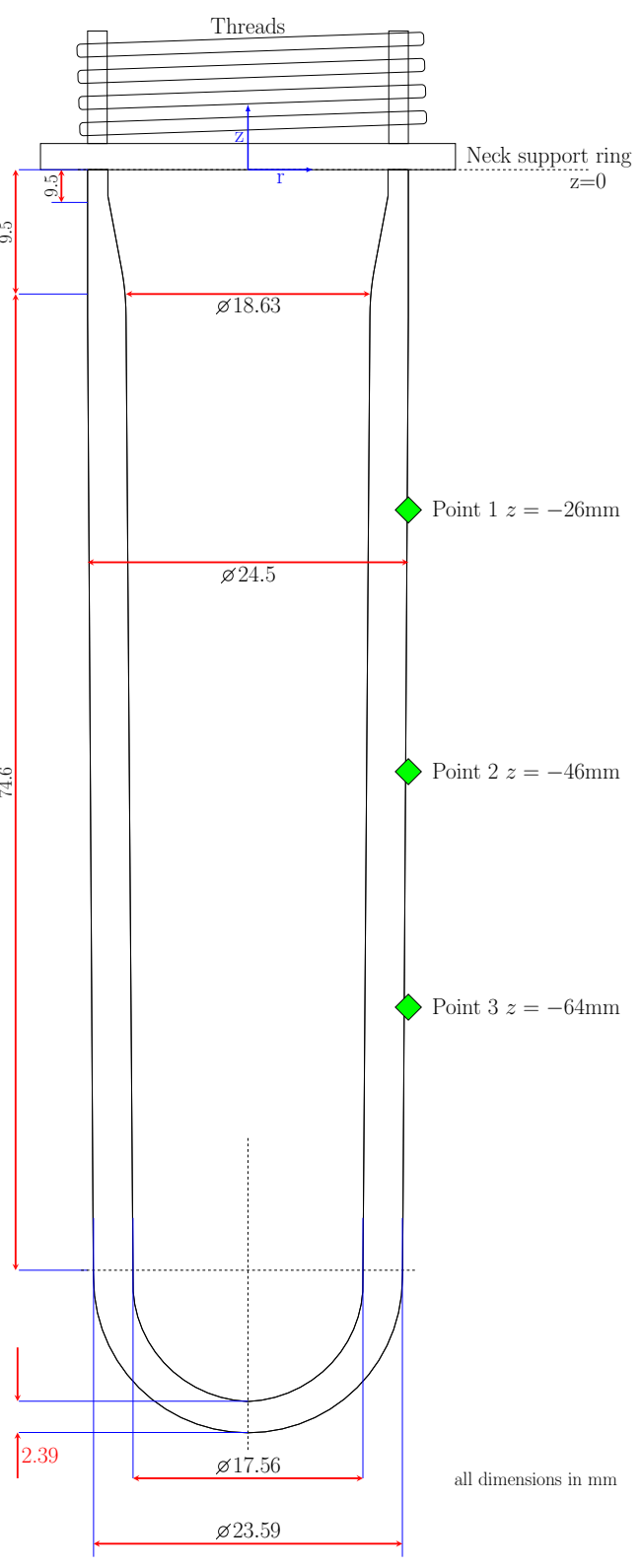

Figure 15: 27g preform geometry with strain sampling points plotted as diamonds

\subsection{Cavity pressure and flow rate}

Initial simulations of SBM idealised the internal pressure through a direct pressure application [48] into the simulation. However, Menary et al. 49] showed that this would, in general, lead to poor correlation between the experimental and numericallybased pressure application. To solve these issues, an ideal gas law was proposed as an alternative mechanism of pressure development. To construct an ideal gas representation of the loading, the pressuretime curve was recorded for each of the trials to derive the mass flow rate of air. The pressure was measured using a sensor placed in the stretch rod [50]. The pressure-time relationship for the flow rate $\mathrm{N} 2$ is shown in Figure 16, where the following characteristic behaviour is observed:

- Between $(t=0)$ and $\mathbf{A}$ the preform is stiff, and material deformation is small.

- After preform yield $\mathbf{A}$, which is a concept outlined in [46], viscous flow occurs, leading to a large volume increase and subsequently a pressure drop according to the ideal gas law.

- Strain hardening occurs along $(\mathbf{C})$, due to increasing molecular alignment. Subsequently, the pressure begins to rise to the supply line pressure.

These same behaviours are also shown in the results obtained for N5 and N8, Figure 17, although they occur at different times, and pressure levels. Comparing the cavity pressures for N2, N5, and N8 an increasing trend in the peak pressure can be seen. This increase signifies a higher level of stress within the preform, which is predominantly due to 
the higher strain rates imposed by the larger flow rates. These higher strain rates lead to a greater degree of orientation in the polymer, and subsequently a higher level of stress. This increased orientation leads to a faster rate of strain hardening, which results in a steeper pressure gradient post viscous flow, as can be seen in these figures.

\subsubsection{Pressure loading}

To derive the equivalent pressure loading, which is a required input for the simulation, a model of the pressure loading within the cavity has to be developed. Fundamentally, the pressure build-up in the cavity is a result of the mass flow rate of air entering the cavity, which is described by the ideal gas equation as

$$
P_{t}=\frac{M_{t} R T}{V_{t}},
$$

where $P_{t}, M_{t}$ and $V_{t}$ are the pressure, mass and volume at time $t$, respectively. The mass is updated through the following relation,

$$
M_{t}=\int_{0}^{t} \dot{m} d t
$$

where $\dot{m}$ is the mass flow rate. The mass flow rate, $\dot{m}$, is assumed to conform to an isentropic flow law, such that the mass flow rate into the cavity, for the unchoked condition, is given by [51]

$$
\begin{array}{r}
\dot{m}=\frac{C_{D} A_{t} P_{l}}{\sqrt{R T_{L}}}\left(\frac{P_{c}}{P_{l}}\right)^{\frac{1}{\gamma}} \times \\
{\left[\frac{2 \gamma}{\gamma-1}\left(1-\left(\frac{P_{c}}{P_{L}}\right)^{\frac{\gamma-1}{\gamma}}\right)\right]^{\frac{1}{2}}}
\end{array}
$$

and for the choked condition $\left(\frac{P_{c}}{P_{l}}=\left(\frac{2}{\gamma+1}\right)^{\frac{\gamma}{\gamma-1}}\right)$ as

$$
\dot{m}=\frac{C_{D} A_{t} P_{l}}{\sqrt{R T_{L}}} \gamma^{\frac{1}{2}}\left(\frac{2}{\gamma+1}\right)^{\frac{\gamma+1}{2 \gamma-2}}
$$

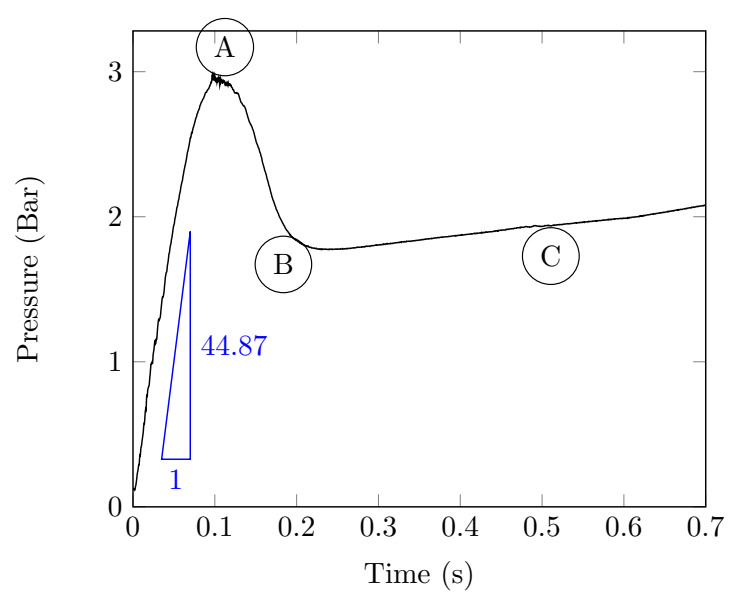

Figure 16: Experimental cavity pressure for N2

In (56), $P_{L}$ and $T_{L}$ are the line pressure and temperature respectively and $P_{c}$ is the cavity pressure. Applying this approach requires the machine to be characterised to determine the effective flow area $\left(C_{D} A\right)$. A technique to perform this was outlined by Yan [47, where the mass flow rate in the choked condition can be found from the initial slope of the pressure-time curve obtained in experiments. In the initial stages of the stretch blow moulding process, the volume can be assumed to be constant. Combining this assumption, with the ideal gas law, and taking the time derivative, yields the mass flow rate,

$$
\dot{m}=\frac{d P}{d t} \frac{V}{R T} .
$$

Through, (57) the choked mass flow rate can be found, which combined with (56) yields the effective flow area $\left(C_{D} A\right)$.

Using this technique, the mass flow can be obtained for each of the experimental trials. The control volume was determined as $V=109000 \mathrm{~mm}^{3}$, based on the volume of the preform cavity below the support ring. The flow rate for N2, Figure 16, the initial slope is given by $d P / d t=44.87 \mathrm{Bar} / \mathrm{s}$, which 
through (57), yields the mass flow rate $\dot{m}=5.60$ $\mathrm{g} / \mathrm{s}$. Repeating this procedure for, N5, and N8 yields $27.98 \mathrm{~g} / \mathrm{s}$ and $54.34 \mathrm{~g} / \mathrm{s}$ respectively, showing the substantial increase in the flow rates imposed.

\subsection{Surface Strain}

The strain history for N5, at the three measurement points is shown in Figure 18 As with the pressure recordings, the strain displays the deformation characteristics of the preform in a number of stages:

- Uniaxial deformation, where the axial strain is increasing, while the hoop strain remains negative. This occurs until preform yield at $\mathbf{A}$.

- After preform yield, both the hoop and axial strains rise rapidly, corresponding to the viscous flow identified in the cavity pressure diagram. In this phase the maximum strain rate is attained.

- Strain hardening begins to occur along $\mathbf{C}$, which causes the strain rate to decrease.

The preform yield is location dependent, with the top point yielding before the bottom point. This produces the characteristic deformation pattern seen in the free blow process, where a bubble forms at the top of the preform and propagates downwards. Within the surface strain results for N2 Figure 19, the preform yield in the hoop direction is delayed with respect to the axial yield point. This leads to sequential deformation, corresponding to axial strain followed by rapid stretching in the hoop direction. This differs from the equibiaxial deformation which is dominant in N5, and N8 Figure 20, and therefore leads to a different material response, which unless accounted for in the material model, can lead to erroneous results at low flow rates.

\subsection{Strain rate}

The maximum hoop and axial strains are shown in Figure 21. As illustrated in the graph, the maximum strain rate increases almost linearly with the mass flow rate. For example, the maximum hoop strain rate increases from $11.87 \mathrm{~s}^{-1}$ at $\mathrm{N} 2$ to $55.68 \mathrm{~s}^{-1}$ for $\mathrm{N} 8$, showing a $369 \%$ increase. As mentioned in the cavity pressure results, the higher strain rates lead to increased molecular orientation, leading to greater cavity pressures. Finally, an important point to note is that the maximum strain rate recorded for $\mathrm{N} 8$ of $55.68 \mathrm{~s}^{-1}$, exceeds the maximum strain rate, of $32 s^{-1}$, used to characterise the material model in [47. This could potentially affect the accuracy of the simulation for this flow rate, which is an issue dealt within the next section.

\section{Stretch blow moulding simulation}

With the implementation of a Lagrangian meshfree method now validated, a simulation of the stretch blow moulding process was constructed by considering the following input parameters:

- Material response

- Loading

- Preform Geometry

In these simulations heat transfer between the stretch rod and preform was not considered. This was judged as an acceptable simplification given that the shape and thickness at the base of the 


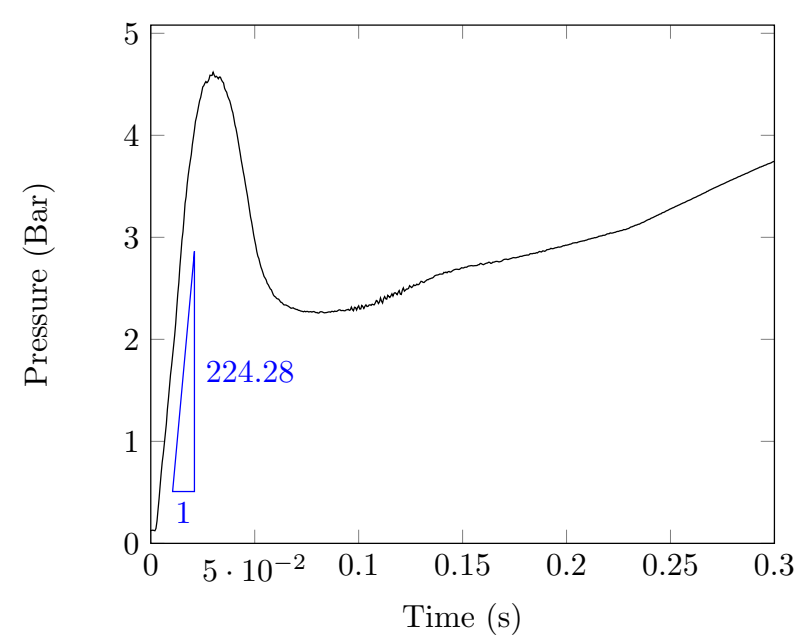

(a)

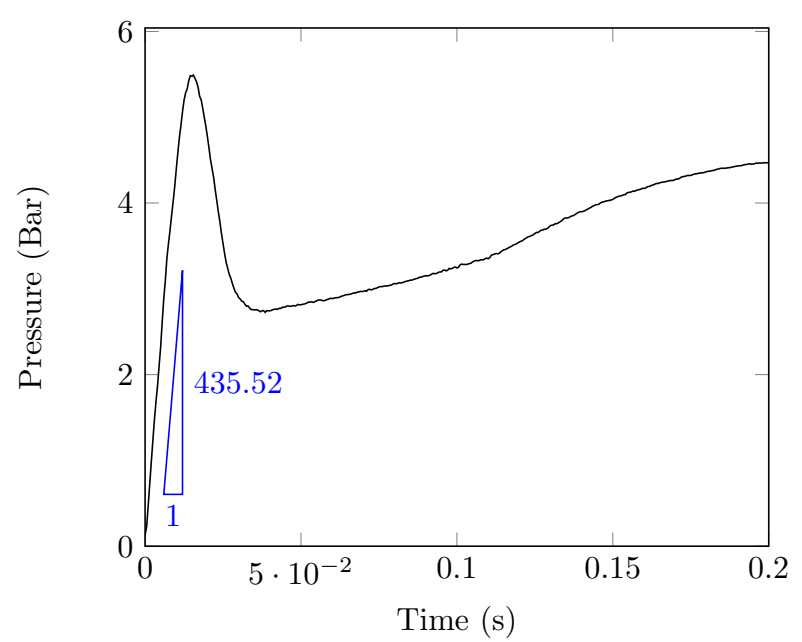

(b)

Figure 17: Experimental cavity pressures for (a) N5 and (b) N8

preform was not investigated in this work. This assumption was also used in the work of Nixon et al. [16].

\subsection{Constitutive Response of PET}

To generate reliable results, a material model that can accurately predict the response of PET is required. A number of different material models have been proposed, including viscoplastic [52], hyper-elastic, 53, and viscoelastic 54. The goal of these models is to capture the response of PET, which is typically dependent on temperature, strain rate and mode of deformation. The objective of this paper is not to provide a detailed discussion of constitutive laws, and instead, we note the frequent use of the physically-based Buckley model [55] in SBM [18, 16, 56.

In this model the stress is decomposed into two contributions, one arising from bond-stretching and the other due to conformational changes within the polymer. This behaviour is captured phe- nomenologically by Figure 22. Based on this bond stress, and conformational stress decomposition, the Cauchy stress, $\boldsymbol{\sigma}$, for this model is defined by:

$$
\begin{aligned}
\boldsymbol{\sigma} & =\boldsymbol{S}^{b}+\boldsymbol{S}^{c}+\boldsymbol{\sigma}^{m}, \\
\boldsymbol{\sigma}^{m} & =k^{b} \ln (J) \mathbf{I},
\end{aligned}
$$

where $\boldsymbol{\sigma}^{m}$ is the mean stress component, $k^{b}$ the bulk modulus of the material, and $J$ the determinant of the deformation gradient. In equation (58a), $S$ is the deviatoric Cauchy stress, defined as

$$
\boldsymbol{S}=\boldsymbol{\sigma}-\frac{1}{3} \operatorname{trace}(\boldsymbol{\sigma})
$$

In the bond-stretching branch the deviatoric bond stress $\boldsymbol{S}^{b}$ is represented by a linear-elastic spring, with shear modulus $G^{b}$, and a viscoelastic element, with relaxation time $\tau$, governed by an Eyring process [57]. The differential representation of this stress is given by,

$$
2 G^{b} \overline{\boldsymbol{D}}=\frac{d \boldsymbol{S}^{b}}{d t}+\frac{\boldsymbol{S}^{b}}{\tau}
$$




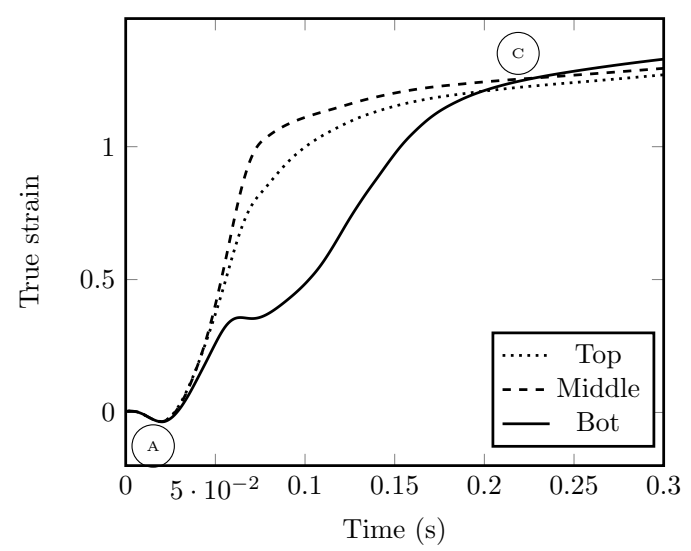

(a)

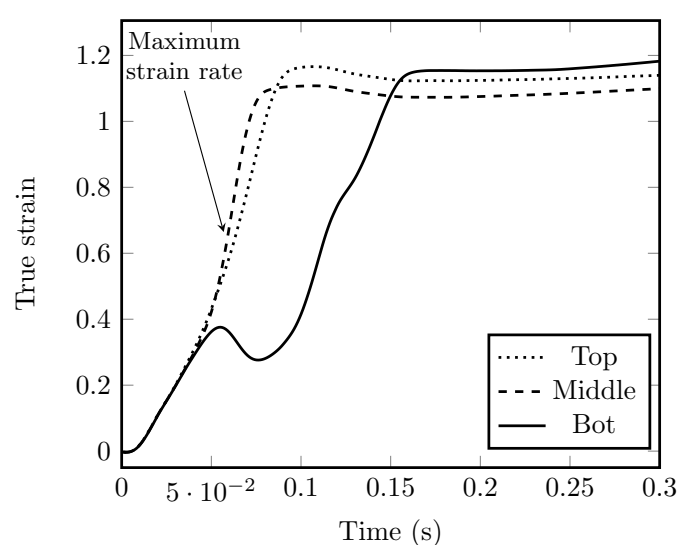

(b)

Figure 18: (N5) Experimental outer wall true strains: (a) hoop and (b) axial

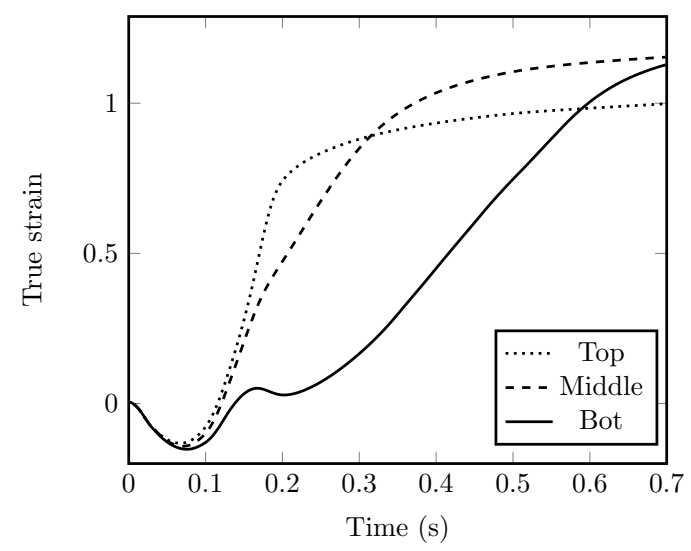

(a)

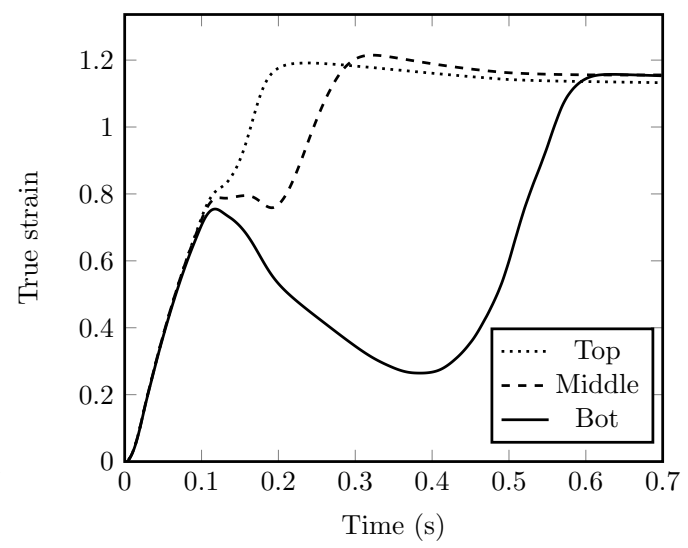

(b)

Figure 19: (N2) Experimental outer wall true strains: (a) hoop and (b) axial 
where $\overline{\boldsymbol{D}}$ is the isochoric deformation gradient, defined by

$$
\overline{\boldsymbol{D}}=\frac{1}{2}\left(\overline{\boldsymbol{L}}+\overline{\boldsymbol{L}}^{T}\right)
$$

and $\overline{\boldsymbol{L}}$ as

$$
\boldsymbol{L}=\frac{d \overline{\boldsymbol{F}}}{d t} \overline{\boldsymbol{F}}^{-1}
$$

In $627, \overline{\boldsymbol{F}}$ is the isochoric part of the deformation gradient given by

$$
\overline{\boldsymbol{F}}=J^{-1 / 3} \boldsymbol{F},
$$

where $J:=\operatorname{det}(\boldsymbol{F})$ is defined as the Jacobian. In the conformational branch the rate of deformation tensor, $\overline{\boldsymbol{D}}$, is decomposed into two terms, $\overline{\boldsymbol{D}}_{s}$ and $\overline{\boldsymbol{D}}_{N}$. The term $\overline{\boldsymbol{D}}_{s}$ represents the slippage between the molecular chains, and induces zero stress, whereas $\overline{\boldsymbol{D}}_{N}$ corresponds to the network deformation, which is responsible for the rubberlike response of the material. The stress-strain response in the conformational branch is governed by the Edwards-Vilgis hyperelastic relation [58, where the principle deviatoric conformation stress components, $S_{k}^{c}$, are given by

$$
S_{k}^{c}=\frac{1}{J} \lambda_{k}^{N} \frac{\partial A^{c}}{\partial \lambda_{k}^{N}}-p \quad(\text { no sum on } \mathrm{k}),
$$

where $\lambda^{N}$ is the deviatoric network stretch, and $p$ is an unknown pressure that occurs due to the constraint $\operatorname{det} \boldsymbol{F}=1$. The strain energy density function, $A^{c}$, for this material is given by,

$$
\begin{aligned}
& A^{c}= \frac{N_{e} K_{B} T}{2}\left[\frac{1+\eta}{1-\alpha^{2} \sum_{i=1}^{3} \lambda_{i}^{N^{2}}} \times\right. \\
& \sum_{i=1}^{3} \frac{\lambda_{i}^{N^{2}}}{1+\eta \lambda_{i}^{N^{2}}}+\sum_{i=1}^{3} \ln \left(1+\eta \lambda_{i}^{N^{2}}\right) \\
&\left.+\ln \left(1-\alpha^{2} \sum_{i=1}^{3} \lambda_{i}^{N^{2}}\right)\right] .
\end{aligned}
$$

In 65, $\alpha$ a measure of in extensibility of the network, $\eta$ the looseness parameter of the entanglements, $K^{B}$ the Boltzmann constant, $T$ the temperature, and $\lambda_{i}^{n}$ the principle network stretches. The entanglement slippage term is responsible for initiating the strain hardening in the material, and is represented by the viscoelastic law

$$
\boldsymbol{D}^{s}=\frac{\boldsymbol{S}^{c}}{\gamma}
$$

where $\gamma$ is viscosity of the slippage dashpot. The network rate of deformation tensor is found from,

$$
\boldsymbol{D}^{N}=\boldsymbol{D}-\boldsymbol{D}^{S} .
$$

The capability of this model in predicting the response of PET in stretch blow moulding is dependent on the material constants used in the model. These constants were obtained in 47, from biaxial stretch experiments at strain rates between $1-32 s^{-1}$ and temperatures between $85^{\circ} \mathrm{C}-115^{\circ} \mathrm{C}$. For completeness, the constants used within this simulation are reproduced in Table 2 .

\subsubsection{Stress updates}

As the constitutive model outlined above is given as a rate formulation, a procedure for performing an objective update is required. A technique commonly applied in explicit methods, due to the small timesteps used, is to perform the stress update in an unrotated configuration. This procedure avoids the use of an objective stress rate, which is often chosen in an ad-hoc manner. In this work we follow the method that is used in an explicit finite element package, Pronto2D [59], and define $\boldsymbol{T}:=\boldsymbol{R}^{T} \boldsymbol{\sigma} \boldsymbol{R}$, as the unrotated stress tensor. The rotation tensor, $\boldsymbol{R}$, is found from the polar decomposition theorem as

$$
\boldsymbol{F}=\boldsymbol{R U} .
$$


The constitutive update procedure based on an unrotated configuration is formulated in terms of the unrotated rate of deformation tensor, $\boldsymbol{d}$, and unrotated stress tensor $\boldsymbol{T}$. For the Buckley material above, the update steps can be summarised as,

$$
\begin{aligned}
\overline{\boldsymbol{d}}_{n+\frac{1}{2}} & =\boldsymbol{R}_{n+1}^{T} \overline{\boldsymbol{D}}_{n+\frac{1}{2}} \boldsymbol{R}_{n+1} \\
\Delta \boldsymbol{T} & =\mathcal{T}\left(\boldsymbol{T}_{n}, \overline{\boldsymbol{d}}_{n+\frac{1}{2}}\right) \\
\boldsymbol{T}_{n+1} & =\boldsymbol{T}_{n}+\Delta \boldsymbol{T} \\
\boldsymbol{\sigma}_{n+1} & =\boldsymbol{R}_{n+1} \boldsymbol{T}_{n+1} \boldsymbol{R}_{n+1}^{T}
\end{aligned}
$$

Bond stress update. The bond stress is updated through analytical integration of the viscoelastic relation 60, assuming a constant relaxation time, $\tau$, over each timestep. This approach is suitable when the timestep is small, which is this case in this explicit meshfree method. Analytical integration of (60), based on the assumptions above, leads to the following update formula

$$
\Delta \boldsymbol{T}^{\boldsymbol{b}}=(1-\exp (\Delta t / \tau))\left(2 G^{b} \tau \overline{\boldsymbol{d}}_{n+\frac{1}{2}}-\boldsymbol{T}_{n}^{b}\right)
$$

Conformational stress update. Once again, due to the small-time step used within the simulations, an explicit stress update procedure is favoured. The conformational stress update procedure requires the split between the network and slippage deformation to be found. The first step of this explicit update is to define the referential true strain tensor, $\overline{\boldsymbol{\epsilon}}$, as the integration of the unrotated rate of deformation tensor,

$$
\overline{\boldsymbol{\epsilon}}_{n+1}=\overline{\boldsymbol{\epsilon}}_{n}+\int_{t_{n}}^{t_{n+1}} \overline{\boldsymbol{d}} d t,
$$

The slippage and network referential true strain are decomposed additively as,

$$
\overline{\boldsymbol{\epsilon}}(t)=\overline{\boldsymbol{\epsilon}}^{s}(t)+\overline{\boldsymbol{\epsilon}}^{N}(t)
$$

Integration of (66), using forward integration, leads to the slippage strain increment of the timestep, given by

$$
\Delta \overline{\boldsymbol{\epsilon}}^{s}=\frac{\boldsymbol{S}_{n}^{c}}{\gamma_{n}} \Delta t
$$

which when combined with the total strain increment $\Delta \overline{\boldsymbol{\epsilon}}=\Delta t \overline{\boldsymbol{d}}_{n+\frac{1}{2}}$, yields the forward update formula for the network strain,

$$
\overline{\boldsymbol{\epsilon}}_{n+1}^{N}=\overline{\boldsymbol{\epsilon}}_{n}^{N}+\Delta t\left(\overline{\boldsymbol{d}}_{n+\frac{1}{2}}-\frac{\boldsymbol{S}_{n}^{c}}{\gamma_{n}}\right) .
$$

From the network referential true strain $\bar{\epsilon}_{n+1}^{N}$, the stretch components $\lambda_{i}$ are found from an eigenvalue decomposition,

$$
\overline{\boldsymbol{\epsilon}}_{i}^{N}=\ln \boldsymbol{\lambda}_{i}^{N}
$$

where $\overline{\boldsymbol{\epsilon}}_{i}^{N}$ are the eigenvalues of $\overline{\boldsymbol{\epsilon}}^{N}$. Finally, the stress response is obtained through the EdwardsVilgis hyperelastic law 64.

\subsection{Loading}

As shown above, the pressure loading in the preform cavity is defined by an ideal gas equation and therefore acts uniformly within the cavity. To update the mass of air in the cavity a forward integration procedure is considered

$$
M(t+\Delta t)=M(t)+\dot{m}(t) \Delta t
$$

where $\dot{m}$ is determined through a function of the pressure ratio in the cavity (55). The preform was subjected to three different choked mass flow rates, which were used to test the ability of the simulation to capture a range of strain rates. The flows rates corresponding to the dimensionless flow indices N2, $\mathrm{N} 5$, and N8 were derived above as $\dot{m}=5.60 \mathrm{~g} / \mathrm{s}$, $27.98 \mathrm{~g} / \mathrm{s}$ and $54.34 \mathrm{~g} / \mathrm{s}$, respectively. The stretch rod speed was prescribed as $1 \mathrm{~m} / \mathrm{s}$, with a maximum travel of $100 \mathrm{~mm}$. 
Table 2: Material Constants

\begin{tabular}{|c|c|}
\hline \multicolumn{2}{|l|}{ Bond-stretching Part } \\
\hline Erying Process & Value \\
\hline Shear activation volume $V_{s}\left(m^{3} \mathrm{~mol}^{-1}\right)$ & $2.814 \times 1$ \\
\hline $\begin{array}{l}\text { Pressure activation volume } V_{p} \\
\left(m^{3} \mathrm{~mol}^{-1}\right)\end{array}$ & $5.262 \times 1$ \\
\hline Reference viscosity $\mu_{0}^{*}(M P a)$ & 1.71 \\
\hline Limiting temperature $T_{\infty}(K)$ & 328.76 \\
\hline Viscosity constant $C^{v}(K)$ & 67.47 \\
\hline Bulk Modulus $K^{b}(M P a)$ & 1800 \\
\hline Shear modulus $G^{b}(M P a)$ & 600 \\
\hline \multicolumn{2}{|l|}{ Conformational Part } \\
\hline Edwards Vilgis & Value \\
\hline Entanglement parameter $\alpha$ & 0.1553 \\
\hline Looseness parameter $\eta$ & 0.001 \\
\hline Entanglement density $N_{s}^{*}\left(m^{-3}\right)$ & $1.81 \times 10$ \\
\hline \multicolumn{2}{|l|}{ Entanglement Slippage } \\
\hline Reference viscosity $\gamma_{0}(\mathrm{MPa})$ & 0.653 \\
\hline Critical stretch gradient $a$ & -0.0356 \\
\hline Critical stretch intercept $b$ & 15.393 \\
\hline
\end{tabular}

\subsection{Preform Temperature}

A single temperature of $105^{\circ} \mathrm{C}$ was used for these simulations. The heating of the preform was conducted using an oil bath to ensure temperature equilibrium. A transportation time of $16 \mathrm{~s}$ was measured between the oil bath, and free-blow machine. To account for the cooling that occurred during this transportation of the preform, the heat transfer simulation outlined by Nixon et al. [16] was utilised. Based on this cooling, an average temper-3 ature of $100.8^{\circ} \mathrm{C}$ was applied throughout the pre${ }_{4}$ form.

\subsection{Geometry}

The geometry of the preform used in these simulations is shown Figure 15. The preform was modelled using an axisymmetric formulation with the geometry idealised by 368 nodes, and 546 stress points shown in Figure 23 along with the Voronoi cells used for SCNI. The domain size was determined by ensuring that at least 3 nodes were within the support of each point, a condition required for the inversion of the moment matrix. The domain size multiplier $d_{\max }$ was kept constant at 2.0 across the whole domain, with a circular support domain used throughout. The critical time-step size was kept constant at $\Delta t=4 \times 10^{-7} \mathrm{~s}$ throughout the simulation.

For these simulations, an $11 \mathrm{~mm}$ stretch rod was modelled as a rigid surface. Contact between the preform and the rigid stretch-rod was modelled using the deformable-to-rigid surface contact algorithm used in Pronto2D [59]. In this method, a predicted kinematic state obtained from the explicit scheme, is modified at the end of each timestep to 
correct for any non-physical penetration between the two bodies.

\section{Results and discussion}

In this section a comparison between the simulation results, and those obtained by experiment is made. To compare the strains, three points on the outside wall of the preform are considered: a bottom point at $y=-64 \mathrm{~mm}$, a top point at $y=-26$ $\mathrm{mm}$ and middle point at $y=-46 \mathrm{~mm}$, as shown in Figure 15. To determine a numerical measure of the error, a normalised root mean square error indicator (NRMSE) is used, defined by

$$
\% N R M S E=\frac{100}{y_{\max }-y_{\min }} \times \sqrt{\frac{\sum_{i}^{n}\left(\bar{y}_{i}-y_{i}\right)^{2}}{n}},
$$

where $\bar{y}$ is the predicted value and $y$ the value obtained from the experiment.

\subsection{Surface strain}

The true strain components are displayed in a cylindrical coordinate system $(r, z, \theta)$.

The results for the lowest flow rate are shown in Figure 24. The first observation to note is that the hoop strain predictions for both the top and middle points show a very good agreement with the experimental results. The axial strains are underpredicted across all measurement points, and predictions at the bottom measurement point display a poor agreement with the experimental results. The primary reason for this poor accuracy is the sequential deformation which develops as a result of the low flow rate. Within the material model, the constants were obtained under biaxial loading, and therefore the constant width deformation mode has not been accounted for. Furthermore, analysis by Yan and Menary [60] highlighted the difficulties in capturing sequential deformation using the Buckley model. The authors showed that if the initial stretch was large, then the final biaxial predictions were poor compared to the experimental results. The case of constant width loading is replicated within the strain predictions for N2T105, Figure 24, where the sequential nature of the deformation is evidenced by the initial rise in the axial strain, which is then followed by an increase in the hoop strain.

The strain predictions for N5 are shown in Figure 25, where an improvement over the N2 predictions can be seen. As before, the hoop strain predictions display a higher degree of accuracy when compared with the axial strains. Furthermore, the axial strains display a small oscillation, which is likely due to the preform blowing on, and off, the stretch rod. The improved strain predictions observed in this free-blow trial is likely due to the equibiaxial state of deformation imposed by the higher flow rates, as shown in Figure 18 .

The strain predictions for N8 are shown in Figure 26, and once again shows good correlation with the experimental data. As before, and for the same reason, a small oscillation is present in the axial strain predictions. Once more, the higher flow leads to an almost perfect equibiaxial state at the top and middle measurement points, explaining the good predictions.

Across all three flow rates, a lower degree of accuracy was obtained at the bottom measure point, compared with middle and top points. This is primarily due to the unavoidable sequential de- 
formation that occurs at the bottom of the preform.

\subsubsection{NRMSE}

The average normalised root mean square error across the top, middle and bottom measurement points are shown in Figure 27. As expected the lowest flow rate trial N2 leads to the highest errors due to the sequential deformation mode imposed. As the flow rate increases the NMRSE errors drop with the best results obtained for the flow rate N5. The average error for the $\mathrm{N} 8$ flow rate is higher than the error obtained for $N 5$, which is likely due to the higher strain rates observed in the $N 8$ trial. These strain rates exceed the maximum strain rates used to characterise the material model, and hence have not been accounted for in the fitting procedure.

The axial strain was in general predicted to a lower degree of accuracy compared to the hoop strain. A reason for this was mentioned above, where the higher flow rate trials cause the bottom of the preform to blow off the stretch rod. This error is potentially due to the mean stress component, $\sigma^{b}=\kappa \ln (J)$, which has not been accounted for in the numerical fitting of the material model. In previous work using this material model, a shellbased element was used [18, 16, where incompressibility was directly enforced. Within the explicit formulation used in this work, the degree of incompressibility that can be enforced is restricted due to numerical stability concerns.

\subsection{Shape comparison}

The comparison of the bottle shape for the flow rates N5 and N8 are shown in Figure 28, The ex- tracted DIC surface is shown as green, red and blue dots in all cases. For both flow rates, the final bottle shape is in very good agreement with the DIC results. However, as could be seen in the strain results, the intermediate shape shows a few differences, which is especially prevalent at the base, where the largest difference between the profiles can be seen.

The results for the $\mathrm{N} 2$ flow rate are shown in Figure 29. As indicated in the strain results for this flow rate, the shape prediction is not as accurate as those obtained for the higher flow rates.

\subsection{Discussion}

Overall, an explicit element-free Galerkin method has been able to capture the primary trends within the free blow process, with satisfactory accuracies shown throughout, and very accurate final shape predictions, Furthermore, as discussed, these errors can be reduced through the additional fitting of the material model. Moreover, this simulation provides a benefit of using a solid based formulation. Within the finite element method, the large deformation necessitates the application of a shell-based element, where the application of an arbitrary temperature profile is not possible. This through-thickness temperature variation has the potential to improve the accuracy of the simulation, especially when a mould is present.

\section{Conclusion}

In this work, an implementation of the explicit element-free Galerkin method for large deformation loading was developed. Despite the use of a fixed 
reference frame, a high degree of accuracy and stability was obtained in all the numerical problems investigated in this paper.

This explicit element-free Galerkin formulation was validated through the simulation of the stretch blow moulding process, a widely-used polymerforming technique. When this process is simulated using the finite element method, difficulties can arise due to the large deformation present. Within this work, due to the use of a meshfree method, these difficulties were not encountered, and a stable and accurate solution was obtained for the full range of the deformations.

Nodally integrated meshfree methods are particularly attractive due to their relative simplicity, and inherent meshfree nature. Furthermore, the Lagrangian form provides a computational benefit as the associated complexity with meshfree methods, namely the formation of shape functions and boundary condition treatments are restricted to the pre-processing stage. Based on the stability, and a high degree of accuracy shown within the explicit meshfree formulation used here, this method has the potential to be utilised in a wide range of large deformation problems, where finite element simulations encounter difficulties.

\section{Acknowledgements}

The first author would like to thank the Department for the Economy (DFE) for funding this research. Secondly, thanks is given to the polymer processing and modelling group (PPMG) at Queen's University Belfast for assisting with the experimental procedure outlined in this paper.

\section{References}

[1] K. J. Bathe, Finite element procedures, 1st Edition, Prentice Hall, 1996.

[2] G. R. Liu, K. Y. Dai, T. T. Nguyen, A smoothed finite element method for mechanics problems, Computational Mechanics 39 (6) (2006) 859-877. doi: 10.1007/s00466-006-0075-4

[3] Y. Hu, M. Randolph, H-adaptive fe analysis of elastoplastic non-homogeneous soil with large deformation, Computers and Geotechnics 23 (1) (1998) 61 - 83. doi: https://doi.org/10.1016/S0266-352X (98)00012-3 URL http://www.sciencedirect.com/science/ article/pii/S0266352X98000123

[4] L. B. Lucy, A numerical approach to the testing of the fission hypothesis, The Astronomical Journal 82 (1977) 1013. doi:10.1086/112164

[5] R. A. Gingold, J. J. Monaghan, Smoothed particle hydrodynamics: theory and application to non-spherical stars, Monthly Notices of the Royal Astronomical Society 181 (3) (1977) 375-389. doi:10.1093/mnras/181. 3.375

[6] T. Belytschko, Y. Y. Lu, L. Gu, Element-free Galerkin methods International Journal for Numerical Methods in Engineering 37 (2) (1994) 229-256. doi:10.1002/ nme.1620370205

URL https://doi.org/10.1002/nme.1620370205

[7] W. K. Liu, S. Jun, Y. F. Zhang, Reproducing kernel particle methods International Journal for Numerical Methods in Fluids 20 (8-9) (1995) 1081-1106. doi:10. 1002/fld.1650200824 URL https://doi.org/10.1002/fld.1650200824

[8] V. P. Nguyen, T. Rabczuk, S. Bordas, M. Duflot, Meshless methods: A review and computer implementation aspects, Mathematics and Computers in Simulation 79 (3) (2008) 763 - 813. doi:https: //doi.org/10.1016/j.matcom.2008.01.003 URL http://www.sciencedirect.com/science/ article/pii/S0378475408000062

[9] N. T. Nguyen, T. Q. Bui, C. Zhang, T. T. Truong, Crack growth modeling in elastic solids by the extended meshfree galerkin radial point interpolation method, Engineering Analysis with Boundary Elements 44 (2014) 87 - 97. doi:https: 
//doi.org/10.1016/j.enganabound.2014.04.021.

URL http://www.sciencedirect.com/science/ article/pii/S0955799714000988

[10] N. Muthu, S. Maiti, B. Falzon, W. Yan, Crack propagation in non-homogenous materials: Evaluation of mixed-mode sifs, t-stress and kinking angle using a variant of efg method, Engineering Analysis with Boundary Elements 72 (2016) $11-26$. doi:https: //doi.org/10.1016/j.enganabound.2016.07.017. URL http://www.sciencedirect.com/science/ article/pii/S0955799716301850

[11] G. Li, T. Belytschko, Element-free Galerkin method for contact problems in metal forming analysis Engineering Computations 18 (1/2) (2001) 62-78. doi: 10.1108/02644400110365806

URL https://doi.org/10.1108/02644400110365806

[12] P. Lu, G. Zhao, Y. Guan, X. Wu, Bulk metal forming process simulation based on rigid-plastic/viscoplastic element free galerkin method Materials Science and Engineering: A 479 (1) (2008) 197 - 212. doi:https://doi.org/10.1016/j.msea.2007.06.059 URL http://www.sciencedirect.com/science/ article/pii/S0921509307013421

[13] A. Horton, A. Wittek, G. R. Joldes, K. Miller, A meshless total lagrangian explicit dynamics algorithm for surgical simulation International Journal for Numerical Methods in Biomedical Engineering 26 (8) (2010) 977-998. arXiv:https://onlinelibrary.wiley.com/ doi/pdf/10.1002/cnm.1374, doi:10.1002/cnm.1374 URL https://onlinelibrary.wiley.com/doi/abs/10. 1002/cnm. 1374

[14] S. Li, W. K. Liu, Numerical simulations of strain localization in inelastic solids using meshfree methods, International Journal for Numerical Methods in Engineering 48 (9) (2000) 12851309. doi:10.1002/1097-0207(20000730)48:9<1285:: AID-NME825>3.0.CO;2-H

URL https : //doi.org/10.1002/1097-0207(20000730) 48:9<1285: :AID-NME825>3.0.CO;2-H

[15] Smithers PIRA, The Future of PET Packaging to 2021, Tech. rep. (2016).

[16] J. Nixon, G. H. Menary, S. Yan, Finite element simulations of stretch-blow moulding with experimental validation over a broad process window International Journal of Material Forming 10 (5) (2017) 793-809. doi:10.1007/s12289-016-1320-9

URL http://link.springer.com/10.1007/ s12289-016-1320-9

[17] M. Bordival, F. Schmidt, Y. L. Maoult, V. Velay, Optimization of preform temperature distribution for the stretch-blow molding of PET bottles: Infrared heating and blowing modeling Polymer Engineering \& Science 49 (4) (2009) 783-793. doi:10.1002/pen.21296 URL http://doi.wiley.com/10.1002/pen.21296

[18] Z. J. Yang, E. M. A. Harkin-Jones, C. G. Armstrong, G. H. Menary, Finite element modelling of stretchblow moulding of PET bottles using Buckley model: Plant tests and effects of process conditions and material parameters Proceedings of the Institution of Mechanical Engineers, Part E: Journal of Process Mechanical Engineering 218 (4) (2004) 237-250. doi:10.1243/0954408042466990

URL http://journals.sagepub.com/doi/10.1243/ 0954408042466990

[19] ABAQUS, dassault Systèmes, Providence, RI, USA.

[20] G. H. Menary, Modelling injection stretch-blow moulding and the resulting 'in service' performance of pet bottles, Ph.D. thesis, Queen's University Belfast, Belfast, Nothern Ireland (1 2001).

[21] B. Cosson, L. Chevalier, J. Yvonnet, Optimization of the Thickness of PET Bottles during Stretch Blow Molding by Using a Mesh-free (Numerical) Method International Polymer Processing 24 (3) (2009) 223-233. doi:10.3139/217.2215

URL http://www.hanser-elibrary.com/doi/abs/10. $3139 / 217.2215$

[22] J. Yvonnet, F. Chinesta, P. Lorong, D. Ryckelynck, The constrained natural element method (C-NEM) for treating thermal models involving moving interfaces, International Journal of Thermal Sciences 44 (6) (2005) 559-569. doi:10.1016/j.ijthermalsci.2004.12.007 URL http://linkinghub.elsevier.com/retrieve/ pii/S1290072905000347

[23] B. Cosson, L. Chevalier, G. Régnier, Simulation of the stretch blow moulding process: from the modelling of the microstructure evolution to the end-use elastic 
properties of polyethylene terephthalate bottles International Journal of Material Forming 5 (1) (2012) 39-53. doi:10.1007/s12289-010-1010-y.

URL http://link.springer.com/10.1007/ s12289-010-1010-y

[24] T. Belytschko, Y. Y. Lu, L. Gu, Element-free Galerkin methods (1994). doi:10.1002/nme.1620370205

[25] P. Lancaster, K. Salkauskas, Surface generated by moving least square methods, Mathematics of computation 37 (155) (1981) 141-158. doi:10.1090/S0025-5718-1981-0616367-1 URL https://doi.org/10.1090/ S0025-5718-1981-0616367-1

[26] G. R. Liu, Y. T. Gu, An introduction to meshfree methods and their programming, Springer, 2005.

[27] T. Rabczuk, T. Belytschko, S. Xiao, Stable particle methods based on lagrangian kernels Computer Methods in Applied Mechanics and Engineering 193 (12-14) (2004) 1035-1063. doi:10.1016/j.cma.2003.12.005 URL http://ac.els-cdn.com/S0045782504000088/ 1-s2.0-S0045782504000088-main.pdf?_tid= $12 \mathrm{~b} 34 \mathrm{ec} 8-\mathrm{ec} 8 \mathrm{a}-11 \mathrm{e} 6-\mathrm{bb} 08-00000 \mathrm{aab0f} 01 \mathrm{\& acdnat}=$ 1486399024_839001d89cc2feec2bb70fbe65dfa01b

[28] J. S. Chen, S. Yoon, C. T. Wu, Non-linear version of stabilized conforming nodal integration for Galerkin mesh-free methods, International Journal for Numerical Methods in Engineering 53 (12) (2002) 2587-2615. doi: $10.1002 /$ nme.338

URL https://doi.org/10.1002/nme.338

[29] J.-S. Chen, S. Yoon, C.-T. Wu, Non-linear version of stabilized conforming nodal integration for galerkin mesh-free methods International Journal for Numerical Methods in Engineering 53 (12) (2002) 25872615. arXiv:https://onlinelibrary.wiley.com/doi/ pdf/10.1002/nme.338 doi:10.1002/nme.338

URL https://onlinelibrary.wiley.com/doi/abs/10. 1002/nme.338

[30] T. Belytschko, W. Liu, B. Moran, Nonlinear finite elements for continua and structures. 2000, 2000. arXiv:arXiv:1011.1669v3, doi:10.1016/S0065-230X (09) 04001-9

[31] M. A. Puso, J. S. Chen, E. Zywicz, W. Elmer, Meshfree and finite element nodal integration methods, Interna- tional Journal for Numerical Methods in Engineering 74 (3) (2008) 416-446. arXiv:https://onlinelibrary. wiley.com/doi/pdf/10.1002/nme.2181, doi:10.1002/ nme.2181

URL https://onlinelibrary.wiley.com/doi/abs/10. 1002/nme.2181

[32] C. T. Dyka, P. W. Randles, R. P. Ingel, Stress points for tension instability in sph, International Journal for Numerical Methods in Engineering 40 (13) (1997) 2325-2341. doi:10.1002/(SICI) 1097-0207(19970715) 40:13<2325: :AID-NME161>3.0.CO;2-8

[33] J. Sukky, L. W. Kam, B. Ted, Explicit Reproducing Kernel Particle Methods for large deformation problems International Journal for Numerical Methods in Engineering 41 (1) (1998) 137-166. doi:10.1002/(SICI) 1097-0207(19980115) 41:1<137: : AID-NME280>3.0.C0;2-A

URL https://doi.org/10.1002/(SICI) 1097-0207(19980115) 41:1<137::AID-NME280>3.0. $\mathrm{CO} ; 2-\mathrm{A}$

[34] G. R. Joldes, H. Chowdhury, A. Wittek, K. Miller, A new method for essential boundary conditions imposition in explicit meshless methods, Engineering Analysis with Boundary Elements 80 (May) (2017) 94-104. doi:10.1016/j.enganabound.2017.03.011 URL http://dx.doi.org/10.1016/j.enganabound. 2017.03 .011

[35] J.-S. Chen, C.-T. Wu, S. Yoon, Y. You, A stabilized conforming nodal integration for Galerkin mesh-free methods, International Journal for $\mathrm{Nu}$ merical Methods in Engineering 50 (2) (2001) 435466. doi:10.1002/1097-0207(20010120)50:2<435: : AID-NME32>3.0.CD;2-A

URL https://doi .org/10.1002/1097-0207(20010120) 50:2<435: :AID-NME32>3.0.CO;2-A

[36] R. Tatham, Flexible bars.r. frisch-fay. butterworths, london. 1962. 220 pp., The Journal of the Royal Aeronautical Society 67 (627) (1963) 192-192. doi:10.1017/ S0368393100078202

[37] J. M. Gere, S. P. Timoshenko, Mechanics of Materials, 3rd Edition, Springer US, Boston, MA, 1991. doi:10. 1007/978-1-4899-3124-5

[38] K. Mattiasson, Numerical results from large de- 
flection beam and frame problems analysed by means of elliptic integrals International Journal for Numerical Methods in Engineering 17 (1) 145-153. arXiv:https://onlinelibrary.wiley.com/ doi/pdf/10.1002/nme.1620170113 doi:10.1002/nme. 1620170113

URL https://onlinelibrary .wiley.com/doi/abs/10. 1002/nme.1620170113

[39] J. Dolbow, T. Belytschko, Numerical integration of the Galerkin weak form in meshfree methods, Computational Mechanics 23 (3) (1999) 219-230. doi:10.1007/ s004660050403

URL https://doi .org/10.1007/s004660050403

[40] R. S. Rivlin, Large Elastic Deformations of Isotropic Materials. VI. Further Results in the Theory of Torsion, Shear and Flexure Philosophical Transactions of the Royal Society A: Mathematical, Physical and Engineering Sciences 242 (845) (1949) 173-195. doi: 10.1098/rsta.1949.0009

URL http://rsta.royalsocietypublishing.org/cgi/ doi/10.1098/rsta.1949.0009

[41] W. K. Liu, T. Belytschko, J.-S. Chen, Nonlinear versions of flexurally superconvergent elements Computer Methods in Applied Mechanics and Engineering 71 (3) (1988) 241 - 258. doi:https: //doi.org/10.1016/0045-7825(88)90034-5.

URL http://www.sciencedirect.com/science/ article/pii/0045782588900345

[42] J.-S. Chen, M. Hillman, M. Rüter, An arbitrary order variationally consistent integration for galerkin meshfree methods, International Journal for Numerical Methods in Engineering 95 (5) 387418. arXiv:https://onlinelibrary.wiley.com/doi/ pdf/10.1002/nme.4512, doi:10.1002/nme.4512

URL https://onlinelibrary.wiley.com/doi/abs/10. $1002 / \mathrm{nme} .4512$

[43] Q. Duan, X. Li, H. Zhang, B. Wang, X. Gao, Quadratically consistent one-point (qc1) quadrature for meshfree galerkin methods Computer Methods in Applied Mechanics and Engineering 245-246 (2012) 256 - 272. doi:https://doi.org/10.1016/j.cma.2012.07.019 URL http://www.sciencedirect.com/science/ article/pii/S0045782512002411
[44] G. Menary, P. Martin, J. Nixon, S. Yan, Indicate: A novel instrument for preform/bottle characterisation in injection stretch-blow moulding 2017, proceedings of Polymer Process Engineering (PPE17) Conference ; Conference date: 25-07-2017 Through 27-07-2017. URL http://www.polyeng.com/ppe17

[45] S. Yan, G. Menary, J. Nixon, A novel methodology to characterize the constitutive behaviour of polyethylene terephthalate for the stretch blow moulding process, Mechanics of Materials 104 (2017) 93 - 106. doi: https://doi.org/10.1016/j.mechmat.2016.10.006 URL http://www.sciencedirect.com/science/ article/pii/S0167663616304094

[46] J. Nixon, G. H. Menary, S. Yan, Free-stretch-blow investigation of poly(ethylene terephthalate) over a large process window, International Journal of Material Forming 10 (5) (2017) 765-777. doi:10.1007/ s12289-016-1318-3

[47] S. Yan, Modelling the constitutive behaviour of poly(ethylene terephthalate) for strech blow moulding., Ph.D. thesis, Queen's University Belfast, Queen's University Belfast (10 2013).

[48] J. P. McEvoy, C. G. Armstrong, R. J. Crawford, Simulation of the stretch blow molding process of PET bottles Advances in Polymer Technology 17 (4) (1998) 339-352. doi:10.1002/(SICI) 1098-2329(199824)17: 4<339: :AID-ADV5>3.0.CO;2-S

URL https://doi.org/10.1002/(SICI) 1098-2329(199824) $17: 4<339:$ :AID-ADV5>3.0.CO;2-S

[49] G. Menary, C. Tan, C. Armstrong, Y. Salomeia, M. Picard, N. Billon, E. Harkin-Jones, Validating injection stretch-blow molding simulation through free blow trials Polymer Engineering \& Science 50 (5) (2010) 10471057. doi:10.1002/pen.21555 URL http://doi.wiley.com/10.1002/pen.21555

[50] Y. M. Salomeia, G. H. Menary, C. G. Armstrong, Experimental investigation of stretch blow molding, part 1: Instrumentation in an industrial environment Advances in Polymer Technology 32 (S1) (2013) E771E783. arXiv:https://onlinelibrary.wiley.com/doi/ pdf/10.1002/adv.21320, doi:10.1002/adv.21320 URL https://onlinelibrary.wiley.com/doi/abs/10. $1002 / \mathrm{adv} .21320$ 
[51] Y. Cengel, M. Boles, Thermodynamics : an engineering approach, 8th Edition, McGraw-Hill, Boston, MA, 2014.

[52] L. Chevalier, Y. Marco, Identification of a strain induced crystallisation model for pet under uni- and bi-axial loading: Influence of temperature dispersion. Mechanics of Materials 39 (6) (2007) 596 - 609. doi: https://doi.org/10.1016/j.mechmat.2006.09.001 URL http://www.sciencedirect.com/science/ article/pii/S0167663606001153

[53] G. Marckmann, E. Verron, B. Peseux, Finite element analysis of blow-molding and thermoforming using a dynamic explicit procedure Polymer Engineering and Science 41 (3) (2001) 426-439. doi:10.1002/pen.10740 URL https://hal.archives-ouvertes.fr/ hal-01006898

[54] L. Chevalier, Y. Luo, E. Monteiro, G. Menary, On visco-elastic modelling of polyethylene terephthalate behaviour during multiaxial elongations slightly over the glass transition temperature Mechanics of Materials 52 (2012) 103 - 116 . doi:https: //doi.org/10.1016/j.mechmat.2012.05.003

URL http://www.sciencedirect.com/science/ article/pii/S0167663612000889

[55] C. Buckley, Glass-rubber constitutive model for amorphous polymers near the glass transition Polymer $36 \quad$ (17) (1995) 3301-3312. doi:10.1016/0032-3861(95)99429-X

URL http://linkinghub.elsevier.com/retrieve/ pii/003238619599429X

[56] J. Zimmer, M. Stommel, Method for the evaluation of stretch blow molding simulations with free blow trials, IOP Conference Series: Materials Science and Engineering 48 (2013) 012004. doi : 10.1088/1757-899X/48/1/012004

URL http://stacks.iop.org/1757-899X/ $48 / i=1 / a=012004$ ?key=crossref . 29e086eee562e961ed043b2f4934a05a

[57] G. Halsey, J. Howard J. White, H. Eyring, Mechanical properties of textiles, i. Textile Research 15 (9) (1945) 295-311. doi:10.1177/004051754501500901. URL https://doi.org/10.1177/004051754501500901

[58] S. Edwards, T. Vilgis, The effect of entanglements in rubber elasticity, Polymer 27 (4) (1986) 483 - 492. doi: https://doi.org/10.1016/0032-3861(86)90231-4

URL http://www.sciencedirect.com/science/ article/pii/0032386186902314

[59] L. Taylor, D. Flanagan, Pronto 2d: A twodimensional transient solid dynamics program doi:10. 2172/6671798

[60] S. Y. Yan, G. Menary, Modeling the constitutive behaviour of pet for stretch blow mouldingdoi:10.1063/ 1.3589620 


\section{Appendix A: Meshfree pseudo-code}

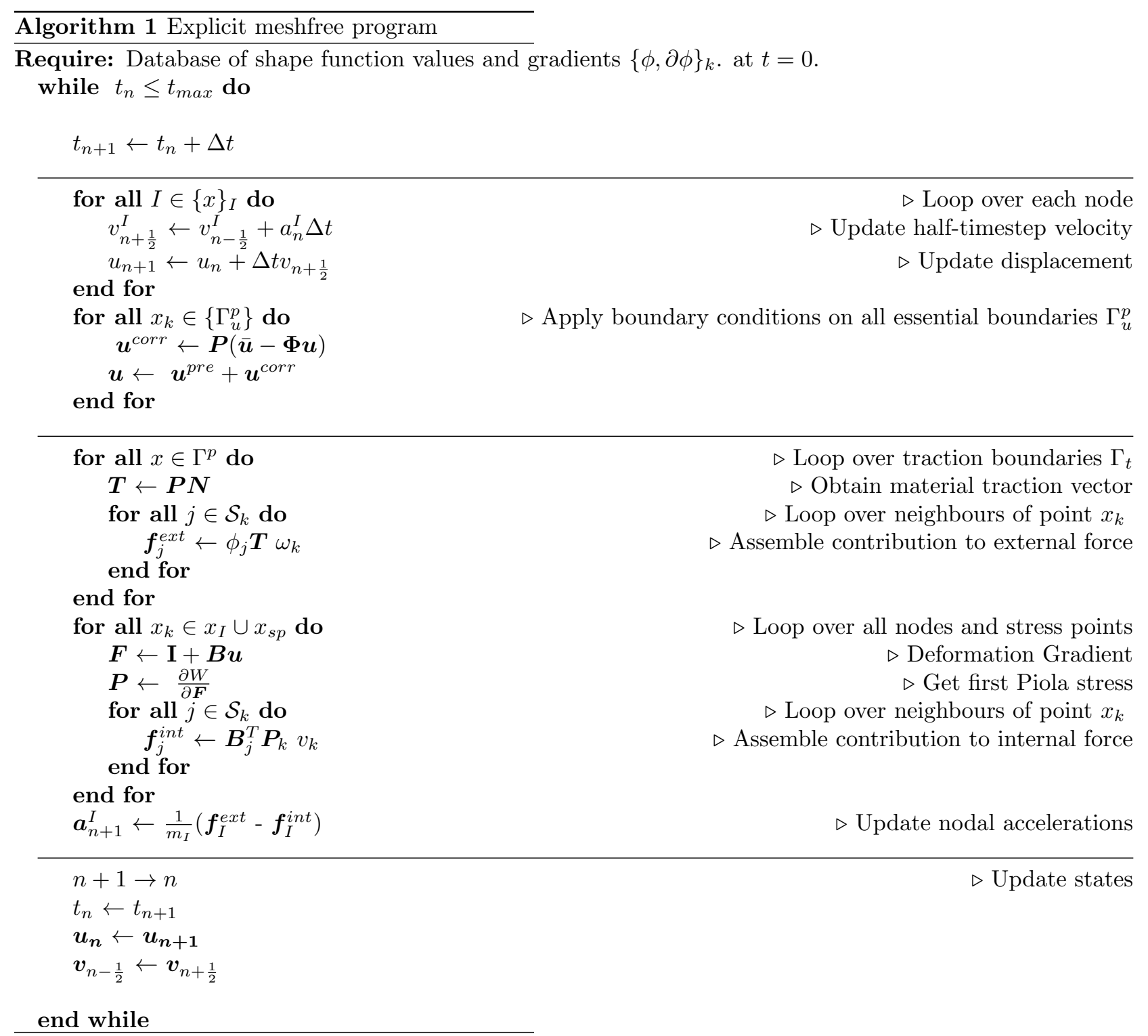




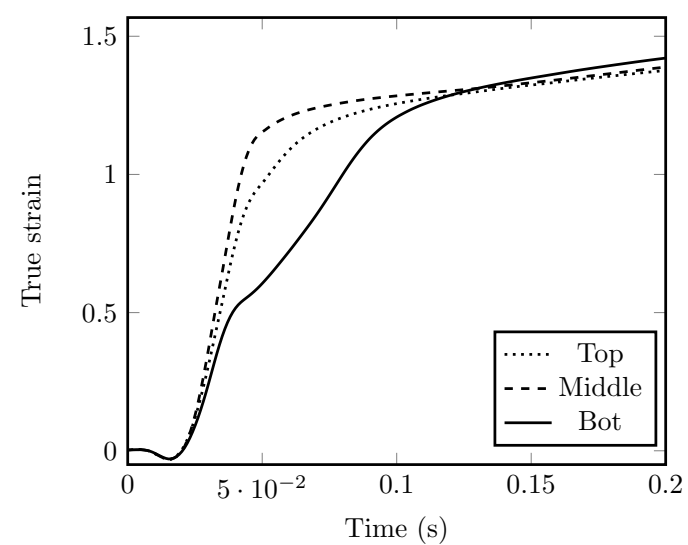

(a)

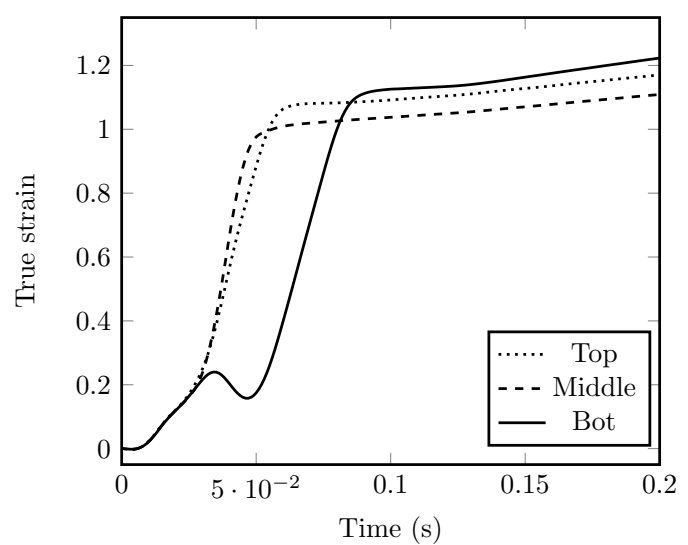

(b)

Figure 20: (N8) Experimental outer wall true strains: (a) hoop and (b) axial 


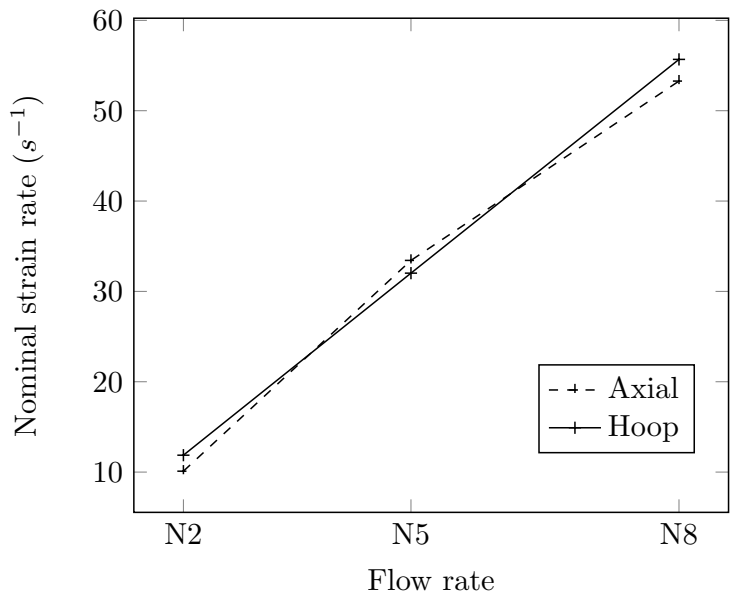

Figure 21: Nominal strain rates in the hoop and axial directions 


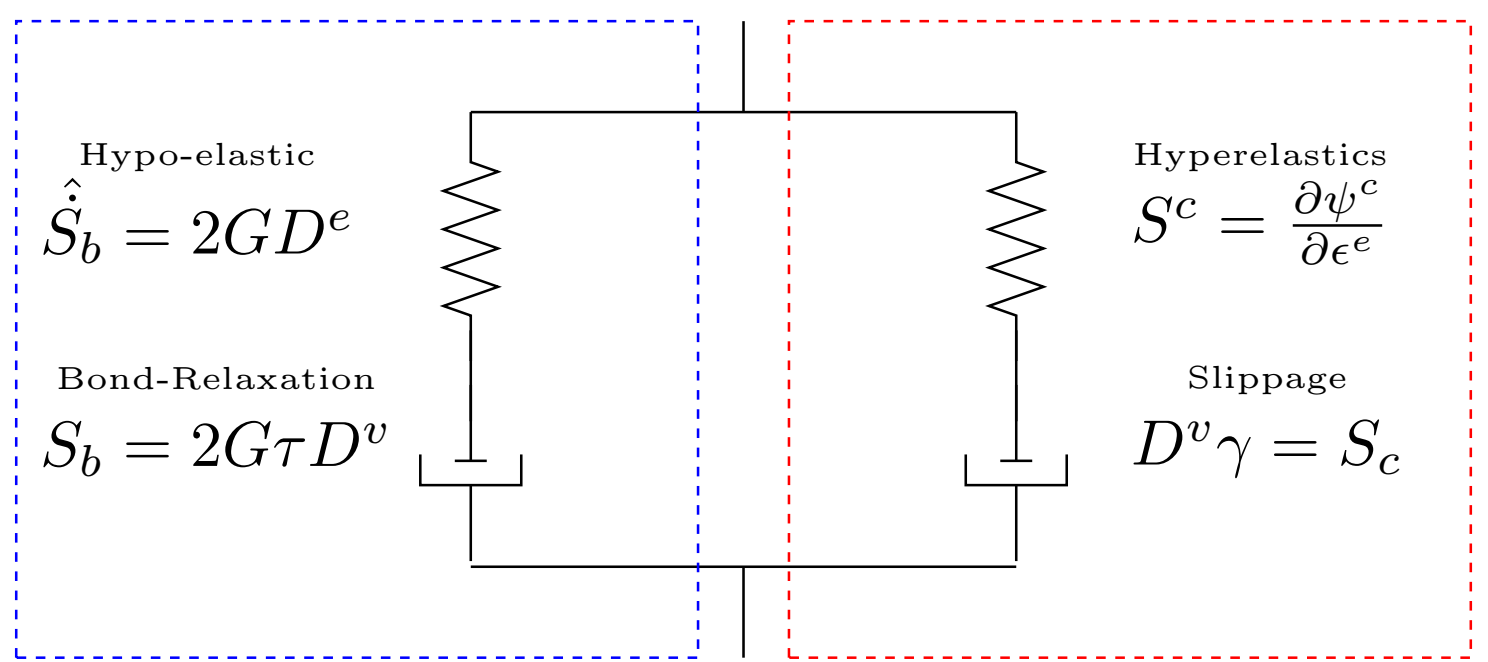

Bond branch

Conformational branch

Figure 22: Buckley material model

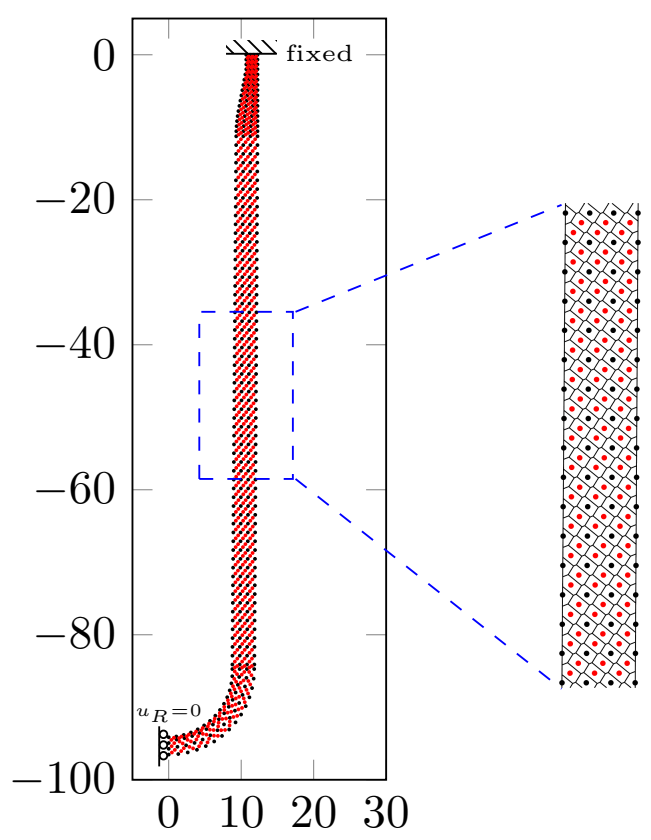

Figure 23: Nodal and stress point distribution: (left) preform discretisation and (right) Voronoi cells used for SCNI 

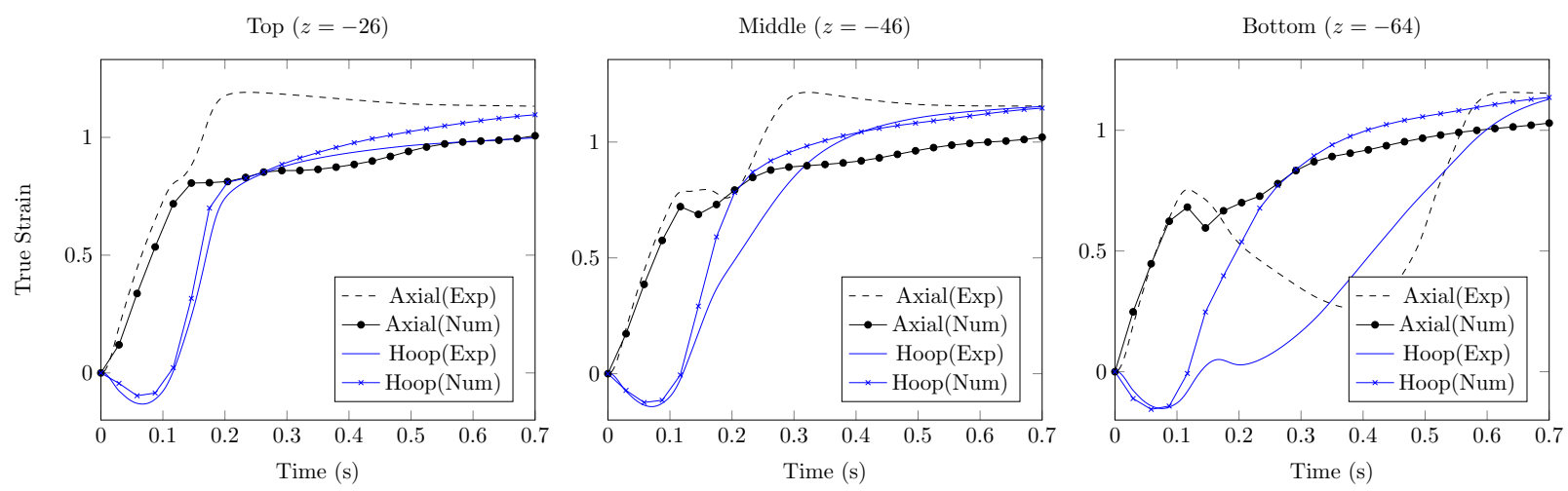

Figure 24: Strain predictions for N2T105
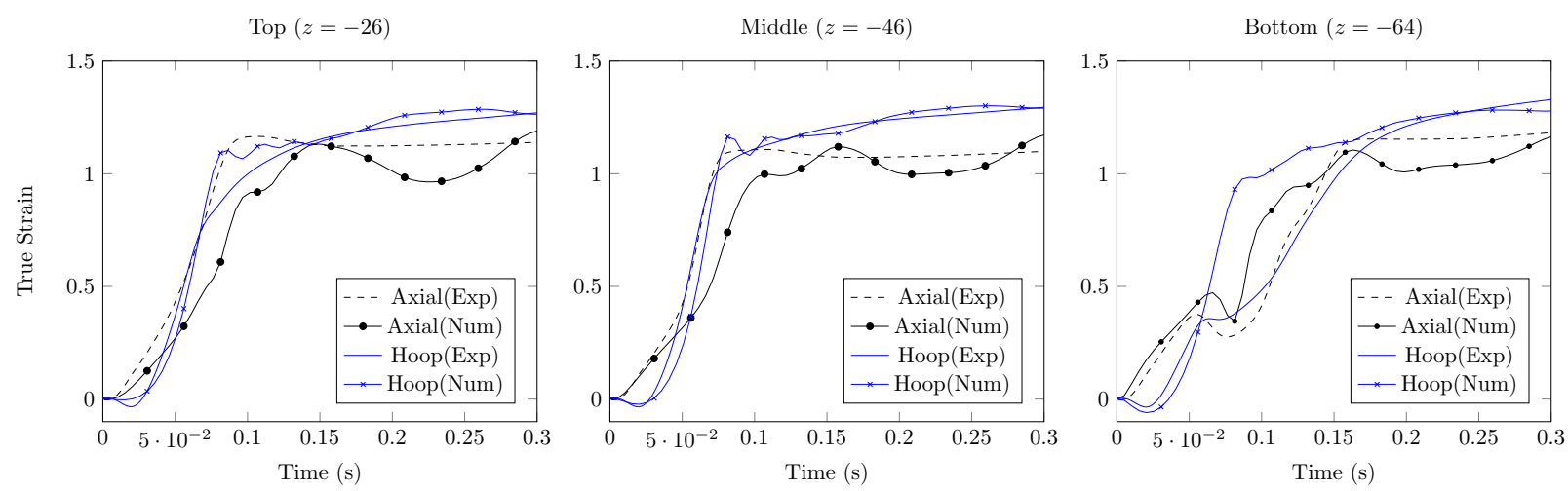

Figure 25: Surface strain for N5T105
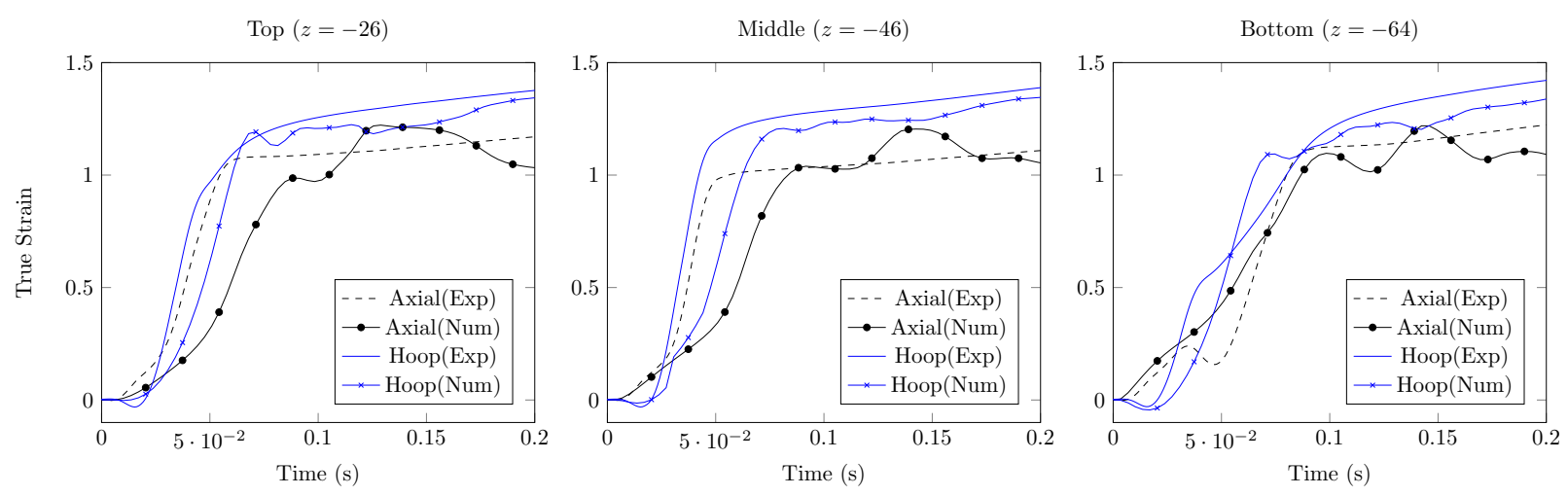

Figure 26: Strain predictions for N8T105 


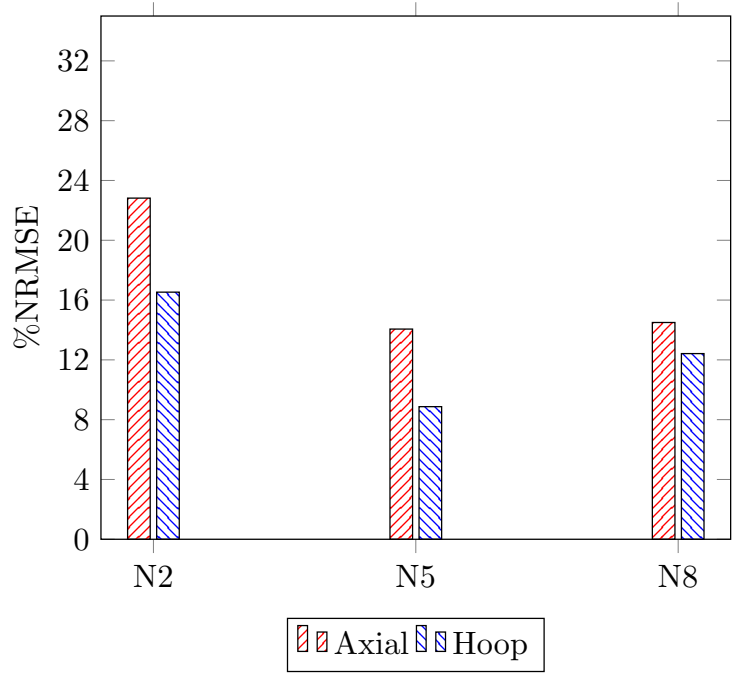

Figure 27: Average NRMSE across the three flow rates 

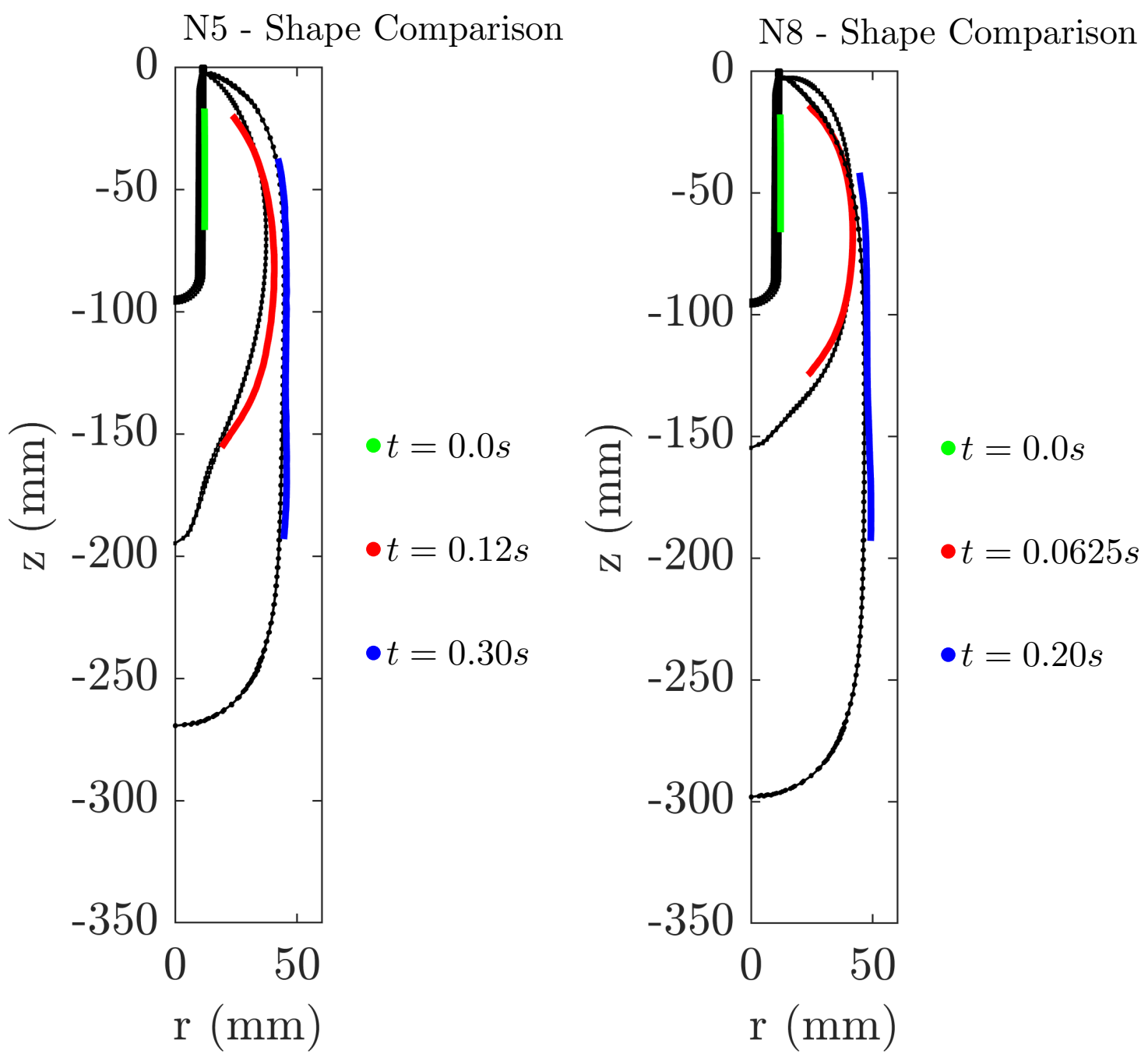

Figure 28: Shape comparison for N5 and N8 


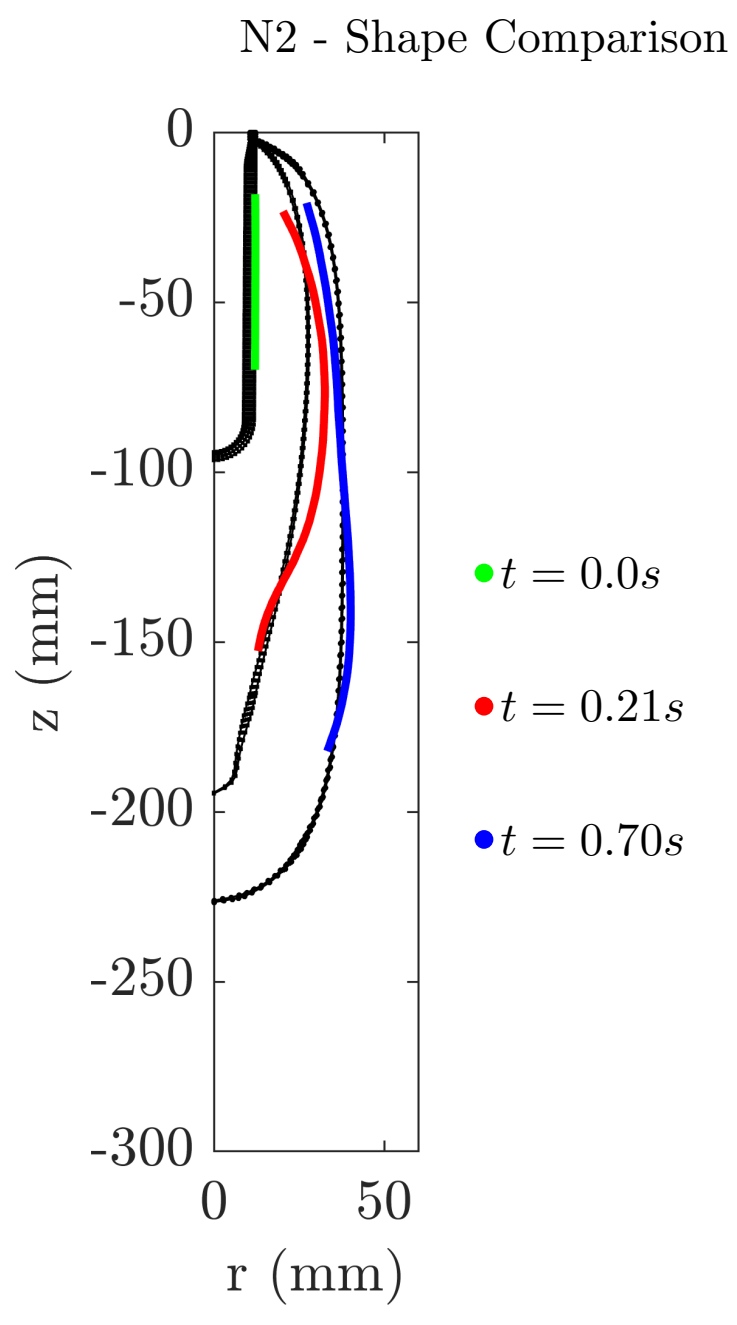

Figure 29: Shape comparison for N2 